\title{
An Efficient Algorithm for the Generalized Foldy-Lax Formulation
}

\author{
Kai Huang, Peijun Li ${ }^{\dagger}$ and Hongkai Zhao ${ }^{\ddagger}$
}

\begin{abstract}
Consider the scattering of a time-harmonic plane wave incident on a two-scale heterogeneous medium, which consists of scatterers that are much smaller than the wavelength and extended scatterers that are comparable to the wavelength. In this work we treat those small scatterers as isotropic point scatterers and use a generalized Foldy-Lax formulation to model wave propagation and capture multiple scattering among point scatterers and extended scatterers. Our formulation is given as a coupled system, which combines the original Foldy-Lax formulation for the point scatterers and the regular boundary integral equation for the extended obstacle scatterers. The existence and uniqueness of the solution for the formulation is established in terms of physical parameters such as the scattering coefficient and the separation distances. Computationally, an efficient physically motivated Gauss-Seidel iterative method is proposed to solve the coupled system, where only a linear system of algebraic equations for point scatterers or a boundary integral equation for a single extended obstacle scatterer is required to solve at each step of iteration. The convergence of the iterative method is also characterized in terms of physical parameters. Numerical tests for the far-field patterns of scattered fields arising from uniformly or randomly distributed point scatterers and single or multiple extended obstacle scatterers are presented.
\end{abstract}

Key words. Foldy-Lax formulation, point scatterers, obstacle scattering, boundary integral equation, Helmholtz equation.

AMS subject classifications. 78A45, 78M15, 65N12

\section{Introduction}

Scattering problems are concerned with how an inhomogeneous medium scatters an incident field. The direct scattering problem is to determine the scattered field from the incident field and the differential equation governing the wave propagation; the inverse scattering problem is to determine the nature of the inhomogeneity, such as location, geometry, and material property, from a knowledge of the scattered field. These problems have played a fundamental role in diverse scientific areas such as radar and sonar, geophysical exploration, medical imaging, near-field and nanooptics. This work is devoted to the modeling and computation of a direct scattering problem,

\footnotetext{
*Department of Mathematics, Florida International University, Miami, FL 33199 (khuang@fiu.edu).

†Department of Mathematics, Purdue University, West Lafayette, IN 47907 (lipeijun@math.purdue.edu). The research was supported in part by NSF grants DMS-0914595, DMS-1042958, and DMS-1151308.

${ }^{\ddagger}$ Department of Mathematics, University of California at Irvine, Irvine, CA 92697 (zhao@math.uci.edu). The research was supported in part by NSF grant DMS-1115698 and ONR grant N00014-11-1-0602.
} 


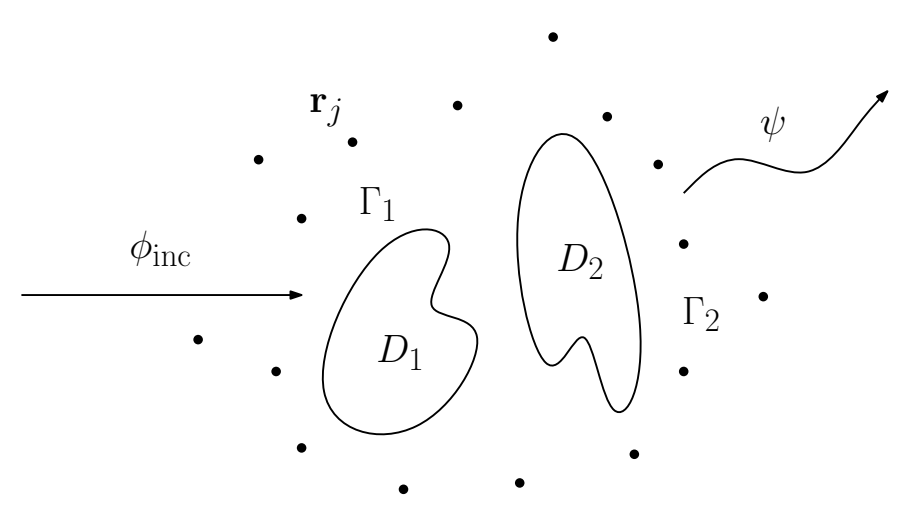

Figure 1: Schematic of problem geometry. The scattered wave $\psi$ is generated from the incidence of a plane wave $\phi_{\text {inc }}$ on the heterogeneous medium consisting a group of point scatterers centered at $\mathbf{r}_{j}$ and an extended obstacle scatterer with possible multiple disjoint components represented by the domain $D_{k}$ with boundary $\Gamma_{k}$.

where the inhomogeneous medium is assumed to be of two-scale nature: it is composed of a group of isotropic point scatterers and an extended obstacle scatterer with possible multiple disjoint components. "Isotropic point scatterer" is a simple but effective model for a scatterer whose size is much smaller than the wavelength of the incident wave so that the scatterer can be represented by a source point within it; "extended" means that the scale of the obstacle scatterer is comparable with the wavelength of the incident field. Precisely, we consider the scattering of a time-harmonic plane wave incident on a heterogeneous medium with both point and extended scatterers, and intend to study the wave propagation and the far-field pattern of the scattered field arising from the interactions between the incident field and all the scatterers, as seen in Figure 1.

The original Foldy-Lax formulation was proposed by Foldy [4] and Lax [8] to describe the multiple scattering of acoustic waves by a group of small isotropic scatterers. This formulation effectively gives the wave fields by solving a self-consistent linear system of algebraic equations for the idealized situation, where the medium is viewed as a collection of isotropic point scatterers. As the size of the scatterers increases to be comparable with the wavelength of the incident field, the original Foldy-Lax formulation will no longer be appropriate to accurately describe the multiple scattering among these scatterers. It is required to consider the effect of the shape of these scatterers on the scattered field. As pointed out in the book by Colton and Kress [3], one of the two basic problems in classical scattering theory is the scattering of time-harmonic acoustic or electromagnetic waves by bounded impenetrable obstacles, which is known as the obstacle scattering problem. Integral equation methods have played an important role in the study of exterior boundary value problems associated with the obstacle scattering. The major advantage of the use of boundary integral equation methods lies in the fact that this approach reduces a problem defined over an unbounded domain to one defined on a bounded domain of lower dimension, i.e., the boundary of the scattering obstacle. We refer to the monograph by Colton and Kress [2] for a comprehensive account of the boundary integral equation methods for solving the obstacle scattering problem.

In many situations it is desirable to develop a model for the simulation of wave propagation in a cluttered medium with embedded extended scatterers, such as in the application of imaging a target in a cluttered environment [10]. Thus it is important to resolve not only the geometry of the extended scatterers but also the effect of the surrounding cluttered medium on the scattered field. Multiple scattering in a multiscale medium presents both mathematical and numerical challenges. In this work, the medium clutter is modeled as a group of isotropic point scatterers and the target is modeled by an extended obstacle scatterer with possible multiple disjoint components. When a 
time-harmonic plane wave is incident on the heterogeneous medium, the scattered waves will be generated from the point scatterers, the extended obstacles and multiple scattering among them. The original Foldy-Lax formulation provides an effective approach to solve the scattering problem from a collection of point scatterers; the method of boundary integral equation has been considered as a desirable choice to solve the obstacle scattering problem. The objective of this work is combine these two methods such that the multiple scattering among all the scatterers can be fully taken into account, without increasing the computational complexity relative to the separated scattering problems especially for multiple extended obstacle scatterers.

Recently, based on the extended-boundary condition method [1], a generalized Foldy-Lax formulation was developed by Huang, Solna, and Zhao [6] for solving the two-dimensional Helmholtz equation in the heterogeneous medium, where the formulation is represented as a series in terms of spherical harmonics and scattering operators. Due to the restriction of the extended-boundary condition method, the formulation in [6] is limited to a single obstacle scatterer. It also assumes that the point scatterers and the obstacle scatterer can be separated by a circle: all the point scatterers are located in the exterior of the circle; the obstacle scatterer is located in the interior of the circle. To overcome the limitations, Huang and Li [5] studied the two-scale multiple scattering problem and proposed a boundary integral equation based generalized Foldy-Lax formulation for the three-dimensional Helmholtz equation for arbitrarily distributed point scatterers. In principle, the formulation in [5] can be used to deal with not only an obstacle scatterer with general geometry but also multiple disjoint obstacle scatterers. However, no discussion is given on how to efficiently solve the generalized Foldy-Lax formulation when the extended obstacle scatterer has more than one component.

To be able to handle more general obstacle scatterers including multiple disjoint components, we re-examine the generalized Foldy-Lax formulation given in [5] and develop an improved formulation, which is more stable with respect to the wavenumber since a uniquely solvable boundary integral equation is incorporated into the coupled system. Sufficient conditions for the existence and uniqueness for the solution of the improved formulation are established in terms of the physical parameters such as the scattering coefficient of the point scatterers and the separation distance between the point scatterers and the extended obstacle scatterers. Computationally, an efficient physically based Gauss-Seidel iterative method is proposed to solve the coupled system, where only a linear system of algebraic equations or a boundary integral equation for a single obstacle is required to solve at each step of iteration. The convergence of the iterative method is also characterized in terms of the physical parameters such as the scattering coefficients and the separation distances.

Although all discussion in this work uses the two-dimensional Helmholtz equation, all the results can be straightforwardly extended to the three-dimensional Helmholtz equation which has a even simpler Green's function with a faster decay in distance.

The outline of the paper is as follows. In Section 2, the original Foldy-Lax formulation is briefly reviewed for the scattered field from the interaction among a group of point scatterers. Based on combined single- and double-layer potential representations, a uniquely solvable boundary integral equation is introduced for the sound-soft obstacle scattering problem in Section 3. Section 4 is devoted to the improved generalized Foldy-Lax formulation: a coupled scattering system is derived from the boundary integral equation approach; the existence and uniqueness for the solution of the improved formulation is established; a physically motivated block Gauss-Seidel iterative method is proposed, and the convergence of the iteration is discussed. In Section 5, the far-field patterns of the scattered fields from original and generalized Foldy-Lax formulations are described. Numerical techniques, particularly the implementation of the uniquely solvable boundary integral equation, are presented in Section 6. Numerical results are shown in Section 7 for various examples of the 
point scatterers and the extended obstacle scatterers. The paper is concluded with comments and directions for future work in Section 8.

\section{Foldy-Lax formulation for point scatterers}

In this section, we briefly introduce the original Foldy-Lax formulation to model the scattering of isotropic point scatterers. We refer to the book by Martin [9] for detailed discussions on the Foldy-Lax formulation.

Consider a collection of $m$ separated isotropic point scatterers, which can be represented by point sources located at $\mathbf{r}_{1}, \ldots, \mathbf{r}_{m}$. Let $\phi_{\text {inc }}$ be the plane incident wave, given explicitly

$$
\phi_{\text {inc }}(\mathbf{r})=e^{\mathrm{i} \kappa \mathbf{r} \cdot \mathbf{d}} \quad \text { in } \mathbb{R}^{2},
$$

where $\mathrm{i}$ is the imaginary unit, $\kappa$ is the wavenumber, and $\mathbf{d}=(\cos \alpha, \sin \alpha) \in \mathbb{S}^{1}$ is the propagation direction defined on the unit circle and $\alpha \in[0,2 \pi]$ is the incident angle. The incident field satisfies the Helmholtz equation

$$
\Delta \phi_{\text {inc }}+\kappa^{2} \phi_{\text {inc }}=0 \quad \text { in } \mathbb{R}^{2} .
$$

The total field is represented as the sum of the incident field and the scattered field

$$
\phi(\mathbf{r})=\phi_{\mathrm{inc}}(\mathbf{r})+\sum_{j=1}^{m} \sigma_{j} \phi_{j} G\left(\mathbf{r}, \mathbf{r}_{j}\right),
$$

where $\sigma_{j}>0$ is referred to as the scattering coefficient for the $j$-th point scatterer, and $G$ the free space Green's function given as

$$
G\left(\mathbf{r}, \mathbf{r}^{\prime}\right)=\frac{\mathrm{i}}{4} H_{0}^{(1)}\left(\kappa\left|\mathbf{r}-\mathbf{r}^{\prime}\right|\right) .
$$

Here $H_{0}^{(1)}$ is the Hankel function of first kind with order zero.

Evaluating (2.3) at $\mathbf{r}_{i}$ and excluding the self-interaction yield a linear system of algebraic equation for $\phi_{j}, j=1, \ldots, m$ :

$$
\phi_{i}=\phi_{\mathrm{inc}}\left(\mathbf{r}_{i}\right)+\sum_{\substack{j=1 \\ j \neq i}}^{m} \sigma_{j} \phi_{j} G\left(\mathbf{r}_{i}, \mathbf{r}_{j}\right),
$$

which is known as the Foldy-Lax formulation. Once (2.4) is solved, the scattered field can be computed as

$$
\psi(\mathbf{r})=\sum_{j=1}^{m} \sigma_{j} \phi_{j} G\left(\mathbf{r}, \mathbf{r}_{j}\right) .
$$

The Foldy-Lax formulation (2.4) can be written in the matrix form

$$
A \phi=\phi_{\text {inc }},
$$

where $\boldsymbol{\phi}=\left(\phi_{1}, \ldots, \phi_{m}\right)^{\top}, \phi_{\text {inc }}=\left(\phi_{\text {inc }}\left(\mathbf{r}_{1}\right), \ldots, \phi_{\text {inc }}\left(\mathbf{r}_{m}\right)\right)^{\top}$, and the $m \times m$ coefficient matrix $A$ is

$$
A=\left[\begin{array}{cccc}
1 & -\sigma_{2} G\left(\mathbf{r}_{1}, \mathbf{r}_{2}\right) & \cdots & -\sigma_{m} G\left(\mathbf{r}_{1}, \mathbf{r}_{m}\right) \\
-\sigma_{1} G\left(\mathbf{r}_{2}, \mathbf{r}_{1}\right) & 1 & \cdots & -\sigma_{m} G\left(\mathbf{r}_{2}, \mathbf{r}_{m}\right) \\
\vdots & \vdots & \ddots & \vdots \\
-\sigma_{1} G\left(\mathbf{r}_{m}, \mathbf{r}_{1}\right) & -\sigma_{2} G\left(\mathbf{r}_{m}, \mathbf{r}_{2}\right) & \cdots & 1
\end{array}\right] .
$$


Given a vector $\mathbf{x}=\left(x_{1}, x_{2}, \ldots, x_{m}\right)^{\top} \in \mathbb{C}^{m}$ and a matrix $B=\left[b_{i j}\right]_{m \times m} \in \mathbb{C}^{m \times m}$, introduce the maximum norms

$$
\|\mathbf{x}\|_{\infty}=\max _{1 \leq j \leq m}\left|x_{i}\right| \text { and }\|B\|_{\infty}=\max _{1 \leq i \leq m} \sum_{j=1}^{m}\left|b_{i j}\right| .
$$

Define the following two physical parameters, maximum scattering strength and the least separation distance, for the group of point scatterers,

$$
\sigma_{\max }=\max _{1 \leq j \leq m} \sigma_{j} \quad \text { and } \quad \rho_{\mathrm{P}}=\min _{1 \leq i<j \leq m}\left|\mathbf{r}_{i}-\mathbf{r}_{j}\right|
$$

They will be used to characterize the criterion for the well-posedness of the Foldy-Lax formulation (2.6).

Theorem 2.1. If $\sigma_{\max } \rho_{\mathrm{P}}^{-1 / 2}$ is sufficiently small, then the coefficient matrix $A$ is invertible and its inverse matrix has the estimate

$$
\left\|A^{-1}\right\|_{\infty} \leq \gamma_{1},
$$

where the constant $\gamma_{1}$ is independent of $\sigma_{j}$ and $\mathbf{r}_{j}$ for $j=1, \ldots, m$.

Proof. Split the matrix $A=I-M$, where

$$
M=\left[\begin{array}{cccc}
0 & \sigma_{2} G\left(\mathbf{r}_{1}, \mathbf{r}_{2}\right) & \cdots & \sigma_{m} G\left(\mathbf{r}_{1}, \mathbf{r}_{m}\right) \\
\sigma_{1} G\left(\mathbf{r}_{2}, \mathbf{r}_{1}\right) & 0 & \cdots & \sigma_{m} G\left(\mathbf{r}_{2}, \mathbf{r}_{m}\right) \\
\vdots & \vdots & \ddots & \vdots \\
\sigma_{1} G\left(\mathbf{r}_{m}, \mathbf{r}_{1}\right) & \sigma_{2} G\left(\mathbf{r}_{m}, \mathbf{r}_{2}\right) & \cdots & 0
\end{array}\right]
$$

Recalling for large argument, we have the following asymptotic behavior for the Hankel function of first kind with order $\mu$ :

$$
H_{\mu}^{(1)}(z)=\sqrt{\frac{2}{\pi z}} e^{\mathrm{i}\left(z-\frac{\pi}{4}-\frac{\mu \pi}{2}\right)}\left[1+O\left(z^{-1}\right)\right]
$$

Using (2.8), we have for well-separated point scatterers that

$$
\left|G\left(\mathbf{r}_{i}, \mathbf{r}_{j}\right)\right|=\frac{1}{4}\left|H_{0}^{(1)}\left(\kappa\left|\mathbf{r}_{i}-\mathbf{r}_{j}\right|\right)\right| \leq \gamma_{0}\left|\mathbf{r}_{i}-\mathbf{r}_{j}\right|^{-1 / 2} \leq \gamma_{0} \rho_{\mathrm{P}}^{-1 / 2}
$$

where $\gamma_{0}$ is a positive constant. Following the matrix norm and (2.9) yield

$$
\|M\|_{\infty} \leq \sigma_{\max } \sum_{\substack{j=1 \\ j \neq i}}^{m}\left|G\left(\mathbf{r}_{i}, \mathbf{r}_{j}\right)\right| \leq m \gamma_{0} \sigma_{\max } \rho_{\mathrm{P}}^{-1 / 2}<1
$$

for sufficiently small $\sigma_{\max } \rho_{\mathrm{P}}^{-1 / 2}$. The above estimate shows that the matrix $A=I-M$ is invertible, and furthermore leads to the estimate

$$
\begin{aligned}
\left\|A^{-1}\right\|_{\infty} & =\left\|(I-M)^{-1}\right\|_{\infty} \leq\left(1-\|M\|_{\infty}\right)^{-1} \\
& \leq\left(1-m \gamma_{0} \sigma_{\max } \rho_{\mathrm{P}}^{-1 / 2}\right)^{-1}=\gamma_{1} .
\end{aligned}
$$


Remark 2.1. As seen from Theorem 2.1, given the number of the point scatterers $m$, if the maximum scattering coefficient $\sigma_{\max }$ is sufficiently small or the separation distance among the point scatterers $\rho_{\mathrm{P}}$ is sufficiently large, then the Foldy-Lax formulation (2.6) has a unique solution. Furthermore, we have from (2.4) that the multiple scattering among the point scatterers is weak and the scattered field can be approximated by the simple summation

$$
\psi(\mathbf{r}) \approx \sum_{j=1}^{m} \sigma_{j} \phi_{\mathrm{inc}}\left(\mathbf{r}_{j}\right) G\left(\mathbf{r}, \mathbf{r}_{j}\right),
$$

which is known as the Born approximation.

\section{Boundary integral equations for extended obstacle scat- terers}

This section is concerned with a brief introduction of the boundary integral equation method for solving the obstacle scattering problem. We assume that the obstacle is represented by the domain $D$ with boundary $\Gamma$, which is the open complement of an unbounded domain of class $C^{2}$, i.e., scattering from more than one component is included in our analysis.

Consider the two-dimensional Helmholtz equation

$$
\Delta \phi+\kappa^{2} \phi=0 \quad \text { in } \mathbb{R}^{2} \backslash \bar{D}
$$

along with the sound-soft boundary condition

$$
\phi=0 \quad \text { on } \Gamma,
$$

where $\phi$ is the total field and $\kappa$ is the wavenumber.

The obstacle is illuminated by the plane incident wave (2.1). The total field $\phi$ consists of the incident field $\phi_{\text {inc }}$ and the scattered field $\psi$ :

$$
\phi=\phi_{\text {inc }}+\psi
$$

It follows from (2.2) and (3.1)-(3.3) that the scattered field satisfies

$$
\Delta \psi+\kappa^{2} \psi=0 \quad \text { in } \mathbb{R}^{2} \backslash \bar{D},
$$

together with the boundary condition

$$
\psi=-\phi_{\text {inc }} \quad \text { on } \Gamma \text {. }
$$

In addition, the scattered field is required to satisfy the Sommerfeld radiation condition

$$
\lim _{\rho \rightarrow \infty} \sqrt{\rho}\left(\frac{\partial \psi}{\partial \rho}-\mathrm{i} \kappa \psi\right)=0, \quad \rho=|\mathbf{r}|,
$$

uniformly for all directions $\hat{\mathbf{r}}=\mathbf{r} /|\mathbf{r}|$.

Based on the Green's representation theorem, it can be shown that the scattered field has the integral representation in terms of the normal derivative of the total field:

$$
\psi(\mathbf{r})=-\int_{\Gamma} G\left(\mathbf{r}, \mathbf{r}^{\prime}\right) \partial_{\mathbf{n}^{\prime}} \phi\left(\mathbf{r}^{\prime}\right) d s\left(\mathbf{r}^{\prime}\right), \quad \mathbf{r} \in D .
$$


To compute the scattered field, it is required to determine $\partial \phi$ on $\Gamma$. We adopt the following uniquely solvable integral equation

$$
\frac{1}{2} \partial_{\mathbf{n}} \phi(\mathbf{r})+\int_{\Gamma}\left[\partial_{\mathbf{n}} G\left(\mathbf{r}, \mathbf{r}^{\prime}\right)-\mathrm{i} \eta G\left(\mathbf{r}, \mathbf{r}^{\prime}\right)\right] \partial_{\mathbf{n}^{\prime}} \phi\left(\mathbf{r}^{\prime}\right) d s\left(\mathbf{r}^{\prime}\right)=\left(\partial_{\mathbf{n}}-\mathrm{i} \eta\right) \phi_{\mathrm{inc}}(\mathbf{r}),
$$

where $\mathbf{n}$ is the unit outward normal with respect to the variable $\mathbf{r}$ and $\eta$ is a nonzero real number and is called the coupling parameter. We refer to Kress [7] for an investigation on the proper choice of the coupling parameter $\eta$ with respect to the condition number of the coefficient matrix for the integral equation.

Given a domain $\Omega$, define

$$
\|u\|_{0, \infty, \Omega}=\sup _{\mathbf{r} \in \Omega}|u(\mathbf{r})| .
$$

For a positive integer $\mu$, introduce a standard Sobolev space

$$
W^{\mu, \infty}(\Omega)=\left\{u \in L_{\mathrm{loc}}^{1}(\Omega):\|u\|_{\mu, \infty, \Omega} \leq \infty\right\}
$$

where the Sobolev norm is

$$
\|u\|_{\mu, \infty, \Omega}=\max _{|\lambda| \leq \mu}\left\|D^{\lambda} u\right\|_{0, \infty, \Omega} .
$$

Due to the unique solvability of the boundary integral equation (3.8), we have the following stability result. The proof may be found in [5].

Theorem 3.1. The unique solution to the boundary integral equation (3.8) depends continuously on the incident field, i.e.,

$$
\left\|\partial_{\mathbf{n}} \phi\right\|_{0, \infty, \Gamma} \leq \gamma_{2}\left\|\phi_{\text {inc }}\right\|_{0, \infty, \Gamma},
$$

where the constant $\gamma_{2}$ depends on $\kappa, \eta$, and $\Gamma$.

Remark 3.1. If the extended obstacle scatterer is consisted of $n$ disjoint components, i.e., $\Gamma=$ $\Gamma_{1} \cup \cdots \cup \Gamma_{n}, \Gamma_{k} \cap \Gamma_{k^{\prime}}=\emptyset, k \neq k^{\prime}$, then the boundary integral equation (3.8) can be written as

$$
\frac{1}{2} \varphi(\mathbf{r})+\sum_{k=1}^{n} \int_{\Gamma_{k}}\left[\partial_{\mathbf{n}} G\left(\mathbf{r}, \mathbf{r}^{\prime}\right)-\mathrm{i} \eta G\left(\mathbf{r}, \mathbf{r}^{\prime}\right)\right] \varphi_{k}\left(\mathbf{r}^{\prime}\right) d s\left(\mathbf{r}^{\prime}\right)=\left(\partial_{\mathbf{n}}-\mathrm{i} \eta\right) \phi_{\mathrm{inc}}(\mathbf{r}),
$$

where $\varphi(\mathbf{r})=\partial_{\mathbf{n}} \phi(\mathbf{r})$ and $\varphi_{k}(\mathbf{r})=\left.\varphi(\mathbf{r})\right|_{\mathbf{r} \in \Gamma_{k}}$.

\section{Generalized Foldy-Lax formulation}

This section is devoted to an improved generalized Foldy-Lax formulation for the multiple scattering involving extended obstacle scatterers and a group of point scatterers. This new coupled system is different from that in [5] since a uniquely solvable boundary integral equation is incorporated, which make it more stable with respect to the wavenumber. The well-posedness of the new formulation is discussed, an efficient block Gauss-Seidel iterative method is proposed for solving the coupled system, and the convergence criteria of the iteration are given in terms of physical parameters. 


\subsection{Coupled scattering system}

Viewing the external field acting on the point scatterers as point sources for the obstacle, we consider the Helmholtz equation

$$
\Delta \phi+\kappa^{2} \phi=-\sum_{j=1}^{m} \sigma_{j} \phi_{j} \delta\left(\mathbf{r}-\mathbf{r}_{j}\right) \quad \text { in } \mathbb{R}^{2} \backslash \bar{D}
$$

along with the sound-soft boundary condition

$$
\phi=0 \quad \text { on } \Gamma
$$

where $\phi_{j}$ is the external field acting on the $j$-th point scatterer. Subtracting the incident field from the total field, we may obtain the equation for the scattered field

$$
\Delta \psi+\kappa^{2} \psi=-\sum_{j=1}^{m} \sigma_{j} \phi_{j} \delta\left(\mathbf{r}-\mathbf{r}_{j}\right) \quad \text { in } \mathbb{R}^{2} \backslash \bar{D} .
$$

The scattered field is also required to satisfy the sound-soft boundary condition (3.5) and the Sommerfeld radiation condition (3.6).

It follows from Green's theorem and the Helmholtz equation (4.3) that the scattered field satisfies

$$
\psi(\mathbf{r})=\sum_{j=1}^{m} \sigma_{j} \phi_{j} G\left(\mathbf{r}, \mathbf{r}_{j}\right)+\int_{\Gamma} \partial_{\mathbf{n}^{\prime}} G\left(\mathbf{r}, \mathbf{r}^{\prime}\right) \psi\left(\mathbf{r}^{\prime}\right) d s\left(\mathbf{r}^{\prime}\right)-\int_{\Gamma} G\left(\mathbf{r}, \mathbf{r}^{\prime}\right) \partial_{\mathbf{n}^{\prime}} \psi\left(\mathbf{r}^{\prime}\right) d s\left(\mathbf{r}^{\prime}\right), \quad \mathbf{r} \in \mathbb{R}^{2} \backslash \bar{D}
$$

and

$$
0=\int_{\Gamma} \partial_{\mathbf{n}^{\prime}} G\left(\mathbf{r}, \mathbf{r}^{\prime}\right) \psi\left(\mathbf{r}^{\prime}\right) d s\left(\mathbf{r}^{\prime}\right)-\int_{\Gamma} G\left(\mathbf{r}, \mathbf{r}^{\prime}\right) \partial_{\mathbf{n}^{\prime}} \psi\left(\mathbf{r}^{\prime}\right) d s\left(\mathbf{r}^{\prime}\right), \quad \mathbf{r} \in D
$$

Similarly, the incident field satisfies

$$
\phi_{\mathrm{inc}}(\mathbf{r})=\int_{\Gamma} G\left(\mathbf{r}, \mathbf{r}^{\prime}\right) \partial_{\mathbf{n}^{\prime}} \phi_{\mathrm{inc}}\left(\mathbf{r}^{\prime}\right) d s\left(\mathbf{r}^{\prime}\right)-\int_{\Gamma} \partial_{\mathbf{n}^{\prime}} G\left(\mathbf{r}, \mathbf{r}^{\prime}\right) \phi_{\text {inc }}\left(\mathbf{r}^{\prime}\right) d s\left(\mathbf{r}^{\prime}\right), \quad \mathbf{r} \in D
$$

and

$$
0=\int_{\Gamma} \partial_{\mathbf{n}^{\prime}} G\left(\mathbf{r}, \mathbf{r}^{\prime}\right) \phi_{\text {inc }}\left(\mathbf{r}^{\prime}\right) d s\left(\mathbf{r}^{\prime}\right)-\int_{\Gamma} G\left(\mathbf{r}, \mathbf{r}^{\prime}\right) \partial_{\mathbf{n}^{\prime}} \phi_{\text {inc }}\left(\mathbf{r}^{\prime}\right) d s\left(\mathbf{r}^{\prime}\right), \quad \mathbf{r} \in \mathbb{R}^{2} \backslash \bar{D} .
$$

Adding (4.4) and (4.7) and using the sound-soft boundary condition (3.5) gives the representation for the scattered field in terms of the total field and the external fields acting on the point scatterers:

$$
\psi(\mathbf{r})=\sum_{j=1}^{m} \sigma_{j} \phi_{j} G\left(\mathbf{r}, \mathbf{r}_{j}\right)-\int_{\Gamma} G\left(\mathbf{r}, \mathbf{r}^{\prime}\right) \partial_{\mathbf{n}^{\prime}} \phi\left(\mathbf{r}^{\prime}\right) d s\left(\mathbf{r}^{\prime}\right), \quad \mathbf{r} \in \mathbb{R}^{2} \backslash \bar{D}
$$

Adding the incident field on both sides of (4.8) yields

$$
\phi(\mathbf{r})=\phi_{\text {inc }}(\mathbf{r})+\sum_{j=1}^{m} \sigma_{j} \phi_{j} G\left(\mathbf{r}, \mathbf{r}_{j}\right)-\int_{\Gamma} G\left(\mathbf{r}, \mathbf{r}^{\prime}\right) \partial_{\mathbf{n}^{\prime}} \phi\left(\mathbf{r}^{\prime}\right) d s\left(\mathbf{r}^{\prime}\right), \quad \mathbf{r} \in \mathbb{R}^{2} \backslash \bar{D} .
$$

To compute the scattered field, it is required to compute the normal derivative of the total field, $\partial_{\mathbf{n}} \phi$, and the external field due to point scatterers, $\phi_{j}, j=1, \ldots, m$. 
give

Evaluating (4.9) on both sides at $\mathbf{r}_{i}$ and excluding the self-interaction for the point scatterers

$$
\phi_{i}=\phi_{\mathrm{inc}}\left(\mathbf{r}_{i}\right)+\sum_{\substack{j=1 \\ j \neq i}}^{m} \sigma_{j} \phi_{j} G\left(\mathbf{r}_{i}, \mathbf{r}_{j}\right)-\int_{\Gamma} G\left(\mathbf{r}_{i}, \mathbf{r}^{\prime}\right) \partial_{\mathbf{n}^{\prime}} \phi\left(\mathbf{r}^{\prime}\right) d s\left(\mathbf{r}^{\prime}\right) .
$$

Using the jump relation for the single-layer and double-layer potentials and taking the normal derivative, we obtain a boundary integral equation on $\Gamma$ :

$$
\begin{array}{r}
\frac{1}{2} \partial_{\mathbf{n}} \phi(\mathbf{r})=\left(\partial_{\mathbf{n}}-\mathrm{i} \eta\right) \phi_{\mathrm{inc}}(\mathbf{r})+\sum_{j=1}^{m} \sigma_{j} \phi_{j} \partial_{\mathbf{n}} G\left(\mathbf{r}, \mathbf{r}_{j}\right) \\
-\int_{\Gamma}\left[\partial_{\mathbf{n}} G\left(\mathbf{r}, \mathbf{r}^{\prime}\right)-\mathrm{i} \eta G\left(\mathbf{r}, \mathbf{r}^{\prime}\right)\right] \partial_{\mathbf{n}^{\prime}} \phi\left(\mathbf{r}^{\prime}\right) d s\left(\mathbf{r}^{\prime}\right) .
\end{array}
$$

It is noticed that the boundary integral over $\Gamma$ vanishes in (4.10) if the extended obstacle is not present, which reduces to the original Foldy-Lax formulation (2.4); the summation over the number of point scatterers vanishes in (4.11) if the point scatterers are not present, which reduces to the regular boundary integral equation (3.8) for solving the obstacle scattering problem. Thus, equations (4.10) and (4.11) form the self-consistent generalized Foldy-Lax formulation to compute the interaction between the extended obstacle and a set of point scatterers.

Remark 4.1. If there are multiple extended scatterers, then the generalized Foldy-Lax formulation can be written as the following coupled system

$$
\begin{gathered}
\phi_{i}=\phi_{\mathrm{inc}}\left(\mathbf{r}_{i}\right)+\sum_{\substack{j=1 \\
j \neq i}}^{m} \sigma_{j} \phi_{j} G\left(\mathbf{r}_{i}, \mathbf{r}_{j}\right)-\sum_{k=1}^{n} \int_{\Gamma_{k}} G\left(\mathbf{r}_{i}, \mathbf{r}^{\prime}\right) \varphi_{k}\left(\mathbf{r}^{\prime}\right) d s\left(\mathbf{r}^{\prime}\right), \\
\frac{1}{2} \varphi(\mathbf{r})=\left(\partial_{\mathbf{n}}-\mathrm{i} \eta\right) \phi_{\mathrm{inc}}(\mathbf{r})+\sum_{j=1}^{m} \sigma_{j} \phi_{j} \partial_{\mathbf{n}} G\left(\mathbf{r}, \mathbf{r}_{j}\right) \\
-\sum_{k=1}^{n} \int_{\Gamma_{k}}\left[\partial_{\mathbf{n}} G\left(\mathbf{r}, \mathbf{r}^{\prime}\right)-\mathrm{i} \eta G\left(\mathbf{r}, \mathbf{r}^{\prime}\right)\right] \varphi_{k}\left(\mathbf{r}^{\prime}\right) d s\left(\mathbf{r}^{\prime}\right),
\end{gathered}
$$

where $\varphi(\mathbf{r})=\partial_{\mathbf{n}} \phi(\mathbf{r})$ and $\varphi_{k}(\mathbf{r})=\left.\varphi(\mathbf{r})\right|_{\mathbf{r} \in \Gamma_{k}}$.

\subsection{Well-posedness of the formulation}

This section is concerned with the well-posedness of the generalized Foldy-Lax formulation (4.10)(4.11). An iterative approach is proposed to deduce criteria which assure the convergence of the iteration, and thus the existence and uniqueness of a solution to the generalized Foldy-Lax formulation.

Theorem 4.1. Given the number of point scatterers $m$ and the arclength of the extended obstacle scatterer $|\Gamma|$, the generalized Foldy-Lax formulation (4.10)-(4.11) has a unique solution if one of the following two conditions holds: (i) $\sigma_{\max }$ is sufficiently small; (ii) $\rho_{\mathrm{P}}$ and $\rho_{\mathrm{PE}}$ are sufficiently large.

Proof. Define the separation distance parameter between the point scatterers and the extended obstacle scatterer

$$
\rho_{\mathrm{PE}}=\min _{1 \leq j \leq m} \min _{\mathbf{r} \in \Gamma}\left|\mathbf{r}_{j}-\mathbf{r}\right|
$$


Define two linear operators that characterizes wave field scattered by the extended scatterers and the point scatterers respectively

$$
M u=\left(\int_{\Gamma} G\left(\mathbf{r}_{1}, \mathbf{r}^{\prime}\right) u\left(\mathbf{r}^{\prime}\right) d s\left(\mathbf{r}^{\prime}\right), \ldots, \int_{\Gamma} G\left(\mathbf{r}_{m}, \mathbf{r}^{\prime}\right) u\left(\mathbf{r}^{\prime}\right) d s\left(\mathbf{r}^{\prime}\right)\right)^{\top}
$$

and

$$
(N \phi)(\mathbf{r})=-\sum_{j=1}^{m} \sigma_{j} \phi_{j} G\left(\mathbf{r}, \mathbf{r}_{j}\right) .
$$

The generalized Foldy-Lax formulation (4.10)-(4.11) can be written as the operator form

$$
\left[\begin{array}{cc}
A & M \\
N & \frac{1}{2} I+K^{\prime}-\mathrm{i} \eta S
\end{array}\right]\left[\begin{array}{l}
\phi \\
\varphi
\end{array}\right]=\left[\begin{array}{c}
\phi_{\text {inc }} \\
f
\end{array}\right]
$$

where $f=\left(\partial_{\mathbf{n}}-\mathrm{i} \eta\right) \phi_{\text {inc }}(\mathbf{r})$, and $S, K^{\prime}$ are the single layer and the adjoint double layer potential operators defined as

$$
\begin{aligned}
(S u)(\mathbf{r}) & =\int_{\Gamma} G\left(\mathbf{r}, \mathbf{r}^{\prime}\right) u\left(\mathbf{r}^{\prime}\right) d s\left(\mathbf{r}^{\prime}\right), \\
\left(K^{\prime} u\right)(\mathbf{r}) & =\int_{\Gamma} \partial_{\mathbf{n}} G\left(\mathbf{r}, \mathbf{r}^{\prime}\right) u\left(\mathbf{r}^{\prime}\right) d s\left(\mathbf{r}^{\prime}\right) .
\end{aligned}
$$

Consider the following iteration: let $\phi^{(0)}=(0, \ldots, 0)^{\top}$ and $\varphi^{(0)}(\mathbf{r})=0$, define $\boldsymbol{\phi}^{(\nu)}$ and $\varphi^{(\nu)}$ for $\nu \geq 1$ by the solutions of the following system of equations

$$
A \phi^{(\nu)}=\phi_{\text {inc }}-M \varphi^{(\nu-1)}
$$

and

$$
\left(\frac{1}{2} I+K^{\prime}-\mathrm{i} \eta S\right) \varphi^{(\nu)}=f-N \phi^{(\nu)} .
$$

Clearly, the vector $\phi^{(\nu)}$ and the function $\varphi^{(\nu)}$ are well-defined due to the invertibility of the matrix $A$ from Theorem 2.1 and the operator $\frac{1}{2} I+K^{\prime}-\mathrm{i} \eta S$ from Theorem 3.1. After $\nu$-th iteration, waves that have been scattered fewer or equal to $\nu$ times between points scatterers and the extended obstacle have been captured.

To prove the convergence of the iteration (4.15) and (4.16), we define the differences of two consecutive approximations

$$
\delta \phi^{(\nu)}=\phi^{(\nu)}-\phi^{(\nu-1)} \quad \text { and } \quad \delta \varphi^{(\nu)}=\varphi^{(\nu)}-\varphi^{(\nu-1)} .
$$

It follows from (4.15) and (4.16) that we have the error equations

$$
A \delta \phi^{(\nu)}=-M \delta \varphi^{(\nu-1)}
$$

and

$$
\left(\frac{1}{2} I+K^{\prime}-\mathrm{i} \eta S\right) \delta \varphi^{(\nu)}=-N \delta \phi^{(\nu)} .
$$

An application of Theorem 2.1 to (4.17) yields

$$
\left\|\delta \phi^{(\nu)}\right\|_{\infty} \leq \gamma_{1}\left\|M \delta \varphi^{(\nu-1)}\right\|_{\infty} .
$$


Using the asymptotic behavior of the Hankel function (2.8) and the definition of the operator $M$, we have

$$
\left\|\delta \phi^{(\nu)}\right\|_{\infty} \leq \gamma_{0} \gamma_{1}|\Gamma| \rho_{\mathrm{PE}}^{-1 / 2}\left\|\delta \varphi^{(\nu-1)}\right\|_{0, \infty, \Gamma},
$$

where $|\Gamma|$ is the arclength of the boundary $\Gamma$ for the extended obstacle scatterer. An application of Theorem 3.1 to (4.18) gives

$$
\left\|\delta \varphi^{(\nu)}\right\|_{0, \infty, \Gamma} \leq \gamma_{2}\left\|N \delta \boldsymbol{\phi}^{(\nu)}\right\|_{0, \infty, \Gamma}
$$

It follows from the asymptotic behavior of the Hankel function (2.8) and the definition of the operator $N$ that

$$
\left\|\delta \varphi^{(\nu)}\right\|_{0, \infty, \Gamma} \leq m \gamma_{0} \gamma_{2} \sigma_{\max } \rho_{\mathrm{PE}}^{-1 / 2}\left\|\delta \phi^{(\nu)}\right\|_{\infty} .
$$

Combining (4.19) and (4.20) gives

$$
\left\|\delta \varphi^{(\nu)}\right\|_{0, \infty, \Gamma} \leq \xi\left\|\delta \varphi^{(\nu-1)}\right\|_{0, \infty, \Gamma} \quad \text { and } \quad\left\|\delta \phi^{(\nu)}\right\|_{\infty} \leq \xi\left\|\delta \phi^{(\nu-1)}\right\|_{\infty},
$$

where

$$
\xi=m \gamma_{0}^{2} \gamma_{1} \gamma_{2}|\Gamma| \sigma_{\max } \rho_{\mathrm{PE}}^{-1}<1
$$

for sufficiently small $\sigma_{\max }$ or sufficiently large $\rho_{\mathrm{PE}}$.

Following the definition of $\varphi^{(\nu)}$, we obtain

$$
\varphi^{(\nu)}=\delta \varphi^{(1)}+\delta \varphi^{(2)}+\cdots+\delta \varphi^{(\nu)}
$$

which gives after using (4.21)

$$
\left\|\varphi^{(\nu)}\right\|_{0, \infty, \Gamma} \leq\left(1+\xi+\xi^{2}+\cdots+\xi^{\nu}\right)\left\|\varphi^{(1)}\right\|_{0, \infty, \Gamma} \leq(1-\xi)^{-1}\left\|\varphi^{(1)}\right\|_{0, \infty, \Gamma} .
$$

Obviously, it follows from the well-posedness of the Foldy-Lax formulation (2.6) and the boundary integral equation (3.8) that $\left\|\varphi^{(1)}\right\|_{0, \infty, \Gamma}<\infty$. Hence, the above estimate indicate that the sequence $\left\{\varphi^{(\nu)}\right\}$ is a bounded sequence, and thus there exists a subsequence, which will be still denoted by $\left\{\varphi^{(\nu)}\right\}$, converge to a limit $\varphi$.

Similarly, we have from the definition of $\delta \phi$ that

$$
\phi^{(\nu)}=\delta \phi^{(1)}+\delta \phi^{(2)}+\cdots+\delta \phi^{(\nu)},
$$

which gives after using (4.21)

$$
\left\|\phi^{(\nu)}\right\|_{\infty} \leq\left(1+\xi+\xi^{2}+\cdots+\xi^{\nu}\right)\left\|\phi^{(1)}\right\|_{\infty} \leq(1-\xi)^{-1}\left\|\phi^{(1)}\right\|_{\infty} .
$$

Since $\left\|\phi^{(1)}\right\|_{\infty}<\infty$, there exists a subsequence, which will be still denoted by $\left\{\phi^{(\nu)}\right\}$, converge to a limit $\phi$.

Next is to prove the uniqueness of the limits. If $\phi^{\prime}=\left(\phi_{1}^{\prime}, \ldots, \phi_{m}^{\prime}\right)^{\top}$ and $\varphi^{\prime}$ are also limits of subsequences $\left\{\phi^{(\nu)}\right\}$ and $\left\{\varphi^{(\nu)}\right\}$, then we have

$$
\begin{array}{r}
\phi_{i}^{\prime}=\phi_{\mathrm{inc}}\left(\mathbf{r}_{i}\right)+\sum_{\substack{j=1 \\
j \neq i}}^{m} \sigma_{j} \phi_{j}^{\prime} G\left(\mathbf{r}_{i}, \mathbf{r}_{j}\right)-\int_{\Gamma} G\left(\mathbf{r}_{i}, \mathbf{r}^{\prime}\right) \varphi^{\prime}\left(\mathbf{r}^{\prime}\right) d s\left(\mathbf{r}^{\prime}\right), \\
\frac{1}{2} \varphi^{\prime}(\mathbf{r})=\left(\partial_{\mathbf{n}}-\mathrm{i} \eta\right) \phi_{\mathrm{inc}}(\mathbf{r})+\sum_{j=1}^{m} \sigma_{j} \phi_{j}^{\prime} \partial_{\mathbf{n}} G\left(\mathbf{r}, \mathbf{r}_{j}\right) \\
-\int_{\Gamma}\left[\partial_{\mathbf{n}} G\left(\mathbf{r}, \mathbf{r}^{\prime}\right)-\mathrm{i} \eta G\left(\mathbf{r}, \mathbf{r}^{\prime}\right)\right] \varphi^{\prime}\left(\mathbf{r}^{\prime}\right) d s\left(\mathbf{r}^{\prime}\right) .
\end{array}
$$


Subtracting (4.22) and (4.23) from (4.10) and (4.11), respectively, and denoting $\delta \boldsymbol{\phi}=\boldsymbol{\phi}-\boldsymbol{\phi}^{\prime}$ and $\delta \varphi=\varphi-\varphi^{\prime}$, we obtain

$$
\begin{gathered}
\delta \phi_{i}-\sum_{\substack{j=1 \\
j \neq i}}^{m} \sigma_{j} \delta \phi_{j} G\left(\mathbf{r}_{i}, \mathbf{r}_{j}\right)=-\int_{\Gamma} G\left(\mathbf{r}_{i}, \mathbf{r}^{\prime}\right) \delta \varphi\left(\mathbf{r}^{\prime}\right) d s\left(\mathbf{r}^{\prime}\right) \\
\frac{1}{2} \delta \varphi(\mathbf{r})+\int_{\Gamma}\left[\partial_{\mathbf{n}} G\left(\mathbf{r}, \mathbf{r}^{\prime}\right)-\mathrm{i} \eta G\left(\mathbf{r}, \mathbf{r}^{\prime}\right)\right] \delta \varphi\left(\mathbf{r}^{\prime}\right) d s\left(\mathbf{r}^{\prime}\right)=\sum_{j=1}^{m} \sigma_{j} \delta \phi_{j} \partial_{\mathbf{n}} G\left(\mathbf{r}, \mathbf{r}_{j}\right) .
\end{gathered}
$$

Regarding (4.24), it follows from Theorem 2.1 that we have

$$
\|\delta \phi\|_{\infty} \leq \gamma_{0} \gamma_{1}|\Gamma| \rho_{\mathrm{PE}}^{-1 / 2}\|\delta \varphi\|_{0, \infty, \Gamma}
$$

Regarding (4.25), using Theorem 3.1 yields

$$
\|\delta \varphi\|_{0, \infty, \Gamma} \leq m \gamma_{0} \gamma_{2} \sigma_{\max } \rho_{\mathrm{PE}}^{-1 / 2}\|\delta \phi\|_{\infty} .
$$

Combining above two estimates gives

$$
\|\delta \phi\|_{\infty} \leq \xi\|\delta \phi\|_{\infty} \quad \text { and } \quad\|\delta \varphi\|_{0, \infty, \Gamma} \leq \xi\|\delta \varphi\|_{0, \infty, \Gamma} .
$$

The uniqueness of the solutions follow from $\delta \phi=0$ and $\delta \varphi=0$ since $\xi<1$ for either sufficiently small $\sigma_{\max }$ or sufficiently large $\rho_{\mathrm{PE}}$.

Remark 4.2. The conditions in Theorem (4.1) are sufficient but may not be necessary for the well-posedness of the generalized Foldy-Lax formulation (4.10)-(4.11).

\subsection{Block Gauss-Seidel iterative method}

In this section, we propose an efficient block Gauss-Seidel iterative method to numerically solve the generalized Foldy-Lax formulation (4.12)-(4.13), especially when there are multiple obstacles, and deduce some criteria to characterize the convergence of the proposed iterative method.

Theorem 4.2. Given the number of point scatterers $m$ and the arclength of the extended obstacle $|\Gamma|$, the block Gauss-Seidel iteration (4.27)-(4.28) is convergent if one of the following two conditions holds: (i) $\sigma_{\max }$ is sufficiently small and $\rho_{\mathrm{E}}$ is sufficiently large; (ii) $\rho_{\mathrm{P}}, \rho_{\mathrm{PE}}$, and $\rho_{\mathrm{E}}$ are sufficiently large.

Proof. Denote two linear operators over the boundary $\Gamma_{k}$ :

$$
M_{k} u=\left(\int_{\Gamma_{k}} G\left(\mathbf{r}_{1}, \mathbf{r}^{\prime}\right) u\left(\mathbf{r}^{\prime}\right) d s\left(\mathbf{r}^{\prime}\right), \ldots, \int_{\Gamma_{k}} G\left(\mathbf{r}_{m}, \mathbf{r}^{\prime}\right) u\left(\mathbf{r}^{\prime}\right) d s\left(\mathbf{r}^{\prime}\right)\right)^{\top}
$$

and

$$
\left(N_{k} \boldsymbol{\phi}\right)(\mathbf{r})=-\left.\sum_{j=1}^{m} \sigma_{j} \phi_{j} G\left(\mathbf{r}, \mathbf{r}_{j}\right)\right|_{\mathbf{r} \in \Gamma_{k}}
$$

Recall the single-layer and adjoint double-layer potential operators defined on the boundary $\Gamma_{k}$ :

$$
\begin{aligned}
\left(S_{k} u\right)(\mathbf{r}) & =\int_{\Gamma_{k}} G\left(\mathbf{r}, \mathbf{r}^{\prime}\right) u\left(\mathbf{r}^{\prime}\right) d s\left(\mathbf{r}^{\prime}\right) \\
\left(K_{k}^{\prime} u\right)(\mathbf{r}) & =\int_{\Gamma_{k}} \partial_{\mathbf{n}} G\left(\mathbf{r}, \mathbf{r}^{\prime}\right) u\left(\mathbf{r}^{\prime}\right) d s\left(\mathbf{r}^{\prime}\right) .
\end{aligned}
$$


The generalized Foldy-Lax formulation (4.12)-(4.13) can be written as

$$
\left[\begin{array}{ccccc}
A & M_{1} & M_{2} & \cdots & M_{n} \\
N_{1} & \frac{1}{2} I+K_{1}^{\prime}-\mathrm{i} \eta S_{1} & K_{2}^{\prime}-\mathrm{i} \eta S_{2} & \cdots & K_{n}^{\prime}-\mathrm{i} \eta S_{n} \\
N_{2} & K_{1}^{\prime}-\mathrm{i} \eta S_{1} & \frac{1}{2} I+K_{2}^{\prime}-\mathrm{i} \eta S_{2} & \cdots & K_{n}^{\prime}-\mathrm{i} \eta S_{n} \\
\vdots & \vdots & \vdots & \ddots & \vdots \\
N_{n} & K_{1}^{\prime}-\mathrm{i} \eta S_{1} & K_{2}^{\prime}-\mathrm{i} \eta S_{2} & \vdots & \frac{1}{2} I+K_{n}^{\prime}-\mathrm{i} \eta S_{n}
\end{array}\right]\left[\begin{array}{c}
\phi \\
\varphi_{1} \\
\varphi_{2} \\
\vdots \\
\varphi_{n}
\end{array}\right]=\left[\begin{array}{c}
\phi_{\text {inc }} \\
f_{1} \\
f_{2} \\
\vdots \\
f_{n}
\end{array}\right]
$$

where $f_{k}(\mathbf{r})=\left.\left(\partial_{\mathbf{n}}-\mathrm{i} \eta\right) \phi_{\text {inc }}(\mathbf{r})\right|_{\mathbf{r} \in \Gamma_{k}}=\left.f(\mathbf{r})\right|_{\mathbf{r} \in \Gamma_{k}}, k=1, \ldots, n$. A block Gauss-Seidel method for the solution of (4.26) reads: Let $\boldsymbol{\phi}^{(0)}=(0, \ldots, 0)^{\top}$ and $\varphi_{k}^{(0)}(\mathbf{r})=0$ for $k=1, \ldots, n$, define $\boldsymbol{\phi}^{(\nu)}$ and $\varphi_{k}^{(\nu)}$ for $\nu \geq 1$ by the solutions of the following system of equations

$$
A \phi^{(\nu)}=\phi_{\text {inc }}-\sum_{k=1}^{n} M_{k} \varphi_{k}^{(\nu-1)}
$$

and

$$
\left(\frac{1}{2} I+K_{k}^{\prime}-\mathrm{i} \eta S_{k}\right) \varphi_{k}^{(\nu)}=f_{k}-N_{k} \phi^{(\nu)}-\sum_{j=1}^{k-1}\left(K_{j}^{\prime}-\mathrm{i} \eta S_{j}\right) \varphi_{j}^{(\nu)}-\sum_{j=k+1}^{n}\left(K_{j}^{\prime}-\mathrm{i} \eta S_{j}\right) \varphi_{j}^{(\nu-1)} .
$$

Next we analyze the convergence of the block Gauss-Seidel iteration (4.27)-(4.28). Let $\boldsymbol{\phi}$ and $\varphi_{k}$ for $k=1, \ldots, n$ be the solution of the generalized Foldy-Lax formulation (4.12)-(4.13). Denote by

$$
\delta \boldsymbol{\phi}^{(\nu)}=\boldsymbol{\phi}-\boldsymbol{\phi}^{(\nu-1)} \quad \text { and } \quad \delta \varphi_{k}^{(\nu)}=\varphi_{k}-\varphi_{k}^{(\nu-1)}
$$

the error between the exact solution and the iterative solution at the step $\nu$. Subtracting (4.27) and (4.28) from (4.12) and (4.13), respectively, we obtain

$$
A \delta \phi^{(\nu)}=-\sum_{k=1}^{n} M_{k} \delta \varphi_{k}^{(\nu-1)}
$$

and

$$
\left(\frac{1}{2} I+K_{k}^{\prime}-\mathrm{i} \eta S_{k}\right) \delta \varphi_{k}^{(\nu)}=-N_{k} \delta \phi^{(\nu)}-\sum_{j=1}^{k-1}\left(K_{j}^{\prime}-\mathrm{i} \eta S_{j}\right) \delta \varphi_{j}^{(\nu)}-\sum_{j=k+1}^{n}\left(K_{j}^{\prime}-\mathrm{i} \eta S_{j}\right) \delta \varphi_{j}^{(\nu-1)} .
$$

Regarding (4.29), an application of Theorem 2.1 yields

$$
\left\|\delta \phi^{(\nu)}\right\|_{\infty} \leq \gamma_{1} \sum_{k=1}^{n}\left\|M_{k} \delta \varphi_{k}^{(\nu-1)}\right\|_{\infty}
$$

Using the asymptotic behavior of the Hankel function (2.8) and the definition of the operator $M_{k}$, we have

$$
\left\|\delta \boldsymbol{\phi}^{(\nu)}\right\|_{\infty} \leq \gamma_{0} \gamma_{1}|\Gamma| \rho_{\mathrm{PE}}^{-1 / 2}\left\|\delta \boldsymbol{\varphi}^{(\nu-1)}\right\|_{0, \infty, \Gamma},
$$

where

$$
\left\|\delta \boldsymbol{\varphi}^{(\nu-1)}\right\|_{0, \infty, \Gamma}=\max _{1 \leq k \leq n}\left\|\delta \varphi_{k}^{(\nu-1)}\right\|_{0, \infty, \Gamma_{k}}
$$


Using Theorem 3.1 for (4.30) gives

$$
\begin{array}{r}
\left\|\delta \varphi_{k}^{(\nu)}\right\|_{0, \infty, \Gamma_{k}} \leq \gamma_{2}\left(\left\|N_{k} \delta \phi^{(\nu)}\right\|_{0, \infty, \Gamma_{k}}+\sum_{j=1}^{k-1}\left\|\left(K_{j}^{\prime}-\mathrm{i} \eta S_{j}\right) \delta \varphi_{j}^{(\nu)}\right\|_{0, \infty, \Gamma_{j}}\right. \\
\left.+\sum_{j=k+1}^{n}\left\|\left(K_{j}^{\prime}-\mathrm{i} \eta S_{j}\right) \delta \varphi_{j}^{(\nu-1)}\right\|_{0, \infty, \Gamma_{j}}\right) .
\end{array}
$$

Using the definition of the linear operator $N_{k}$ gives

$$
\left\|N_{k} \delta \phi^{(\nu)}\right\|_{0, \infty, \Gamma_{k}} \leq\left\|\left.\sum_{j=1}^{m} \sigma_{j} \delta \phi_{j}^{(\nu)} G\left(\mathbf{r}, \mathbf{r}_{j}\right)\right|_{\mathbf{r} \in \Gamma_{k}}\right\|_{0, \infty, \Gamma_{k}} \leq m \gamma_{0} \sigma_{\max } \rho_{\mathrm{PE}}^{-1 / 2}\left\|\delta \boldsymbol{\phi}^{(\nu)}\right\|_{\infty} .
$$

Following the asymptotic behavior of the Hankel functions and the definitions of $S_{j}$ and $K_{j}^{\prime}$ gives

$$
\begin{aligned}
\sum_{j=1}^{k-1}\left\|\left(K_{j}^{\prime}-\mathrm{i} \eta S_{j}\right) \delta \varphi_{j}^{(\nu)}\right\|_{0, \infty, \Gamma_{j}} & \leq(1+\eta) \gamma_{0} \rho_{\mathrm{E}}^{-1 / 2} \sum_{j=1}^{k-1}\left|\Gamma_{j}\right|\left\|\delta \varphi_{j}^{(\nu)}\right\|_{0, \infty, \Gamma_{j}}, \\
& \leq(1+\eta) \gamma_{0}|\Gamma| \rho_{\mathrm{E}}^{-1 / 2}\left\|\delta \boldsymbol{\varphi}^{(\nu)}\right\|_{0, \infty, \Gamma}
\end{aligned}
$$

and

$$
\begin{aligned}
\sum_{j=k+1}^{n}\left\|\left(K_{j}^{\prime}-\mathrm{i} \eta S_{j}\right) \delta \varphi_{j}^{(\nu-1)}\right\|_{0, \infty, \Gamma_{j}} & \leq(1+\eta) \gamma_{0} \rho_{\mathrm{E}}^{-1 / 2} \sum_{j=k+1}^{n}\left|\Gamma_{j}\right|\left\|\delta \varphi_{j}^{(\nu-1)}\right\|_{0, \infty, \Gamma_{j}} \\
& \leq(1+\eta) \gamma_{0}|\Gamma| \rho_{\mathrm{E}}^{-1 / 2}\left\|\delta \varphi^{(\nu-1)}\right\|_{0, \infty, \Gamma},
\end{aligned}
$$

where $\left|\Gamma_{k}\right|$ is the arclength of the boundary curve $\Gamma_{k}$ and the parameter

$$
\rho_{\mathrm{E}}=\min _{\substack{\mathbf{r} \in \Gamma_{k}, \mathbf{r}^{\prime} \in \Gamma_{k}^{\prime} \\ 1 \leq k<k^{\prime} \leq n}}\left|\mathbf{r}-\mathbf{r}^{\prime}\right|
$$

is the separation distance among the different boundary components $\Gamma_{k}$ for the extended obstacle.

Combining (4.32)-(4.35) leads to

$$
\begin{aligned}
\left\|\delta \boldsymbol{\varphi}^{(\nu)}\right\|_{0, \infty, \Gamma} \leq m \gamma_{0} \gamma_{2} \sigma_{\max } \rho_{\mathrm{PE}}^{-1 / 2}\left\|\delta \boldsymbol{\phi}^{(\nu)}\right\|_{\infty} & +(1+\eta) \gamma_{0} \gamma_{2}|\Gamma| \rho_{\mathrm{E}}^{-1 / 2}\left\|\delta \boldsymbol{\varphi}^{(\nu)}\right\|_{0, \infty, \Gamma} \\
& +(1+\eta) \gamma_{0} \gamma_{2}|\Gamma| \rho_{\mathrm{E}}^{-1 / 2}\left\|\delta \boldsymbol{\varphi}^{(\nu-1)}\right\|_{0, \infty, \Gamma} .
\end{aligned}
$$

For a sufficiently large separation distance $\rho_{\mathrm{E}}$, we have $(1+\eta) \gamma_{0} \gamma_{2}|\Gamma| \rho_{\mathrm{E}}^{-1 / 2}<1$. Let

$$
\gamma_{3}=\left[1-(1+\eta) \gamma_{0} \gamma_{2}|\Gamma| \rho_{\mathrm{E}}^{-1 / 2}\right]^{-1}
$$

We obtain from (4.36) that

$$
\left\|\delta \boldsymbol{\varphi}^{(\nu)}\right\|_{0, \infty, \Gamma} \leq m \gamma_{0} \gamma_{2} \gamma_{3} \sigma_{\max } \rho_{\mathrm{PE}}^{-1 / 2}\left\|\delta \boldsymbol{\phi}^{(\nu)}\right\|_{\infty}+(1+\eta) \gamma_{0} \gamma_{2} \gamma_{3}|\Gamma| \rho_{\mathrm{E}}^{-1 / 2}\left\|\delta \boldsymbol{\varphi}^{(\nu-1)}\right\|_{0, \infty, \Gamma}
$$

Combing the estimates (4.31) and (4.37) yield

$$
\left\|\delta \boldsymbol{\varphi}^{(\nu)}\right\|_{0, \infty, \Gamma} \leq \zeta_{1}\left\|\delta \boldsymbol{\varphi}^{(\nu-1)}\right\|_{0, \infty, \Gamma},
$$


where

$$
\zeta_{1}=\left[m \gamma_{0} \gamma_{1} \sigma_{\max } \rho_{\mathrm{PE}}^{-1}+(1+\eta) \rho_{\mathrm{E}}^{-1 / 2}\right] \gamma_{0} \gamma_{2} \gamma_{3}|\Gamma|<1
$$

for sufficiently small $\sigma_{\max }$ and sufficiently large $\rho_{\mathrm{E}}$; or sufficiently large $\rho_{\mathrm{PE}}$ and $\rho_{\mathrm{E}}$. Therefore, we have from (4.38) that

$$
\left\|\delta \boldsymbol{\varphi}^{(\nu)}\right\|_{0, \infty, \Gamma} \leq \zeta_{1}^{\nu-1}\left\|\boldsymbol{\varphi}^{(1)}\right\|_{0, \infty, \Gamma} \rightarrow 0 \quad \text { as } \nu \rightarrow \infty .
$$

Here we have used the fact that $\left\|\varphi^{(1)}\right\|_{0, \infty, \Gamma}<\infty$ due to the well-posedness of the Foldy-Lax formulation (2.6) and the boundary integral equation (3.8).

Similarly, we have from (4.31) and (4.37) that

$$
\left\|\delta \phi^{(\nu)}\right\|_{\infty} \leq \zeta_{2}\left\|\delta \phi^{(\nu-1)}\right\|_{\infty}+\zeta_{3}\left\|\delta \varphi^{(\nu-2)}\right\|_{0, \infty, \Gamma}
$$

where

$$
\zeta_{2}=m \gamma_{0}^{2} \gamma_{1} \gamma_{2} \gamma_{3} \sigma_{\max } \rho_{\mathrm{PE}}^{-1}|\Gamma| \quad \text { and } \quad \zeta_{3}=(1+\eta) \gamma_{0}^{2} \gamma_{1} \gamma_{2} \gamma_{3} \rho_{\mathrm{PE}}^{-1 / 2} \rho_{\mathrm{E}}^{-1 / 2}|\Gamma|
$$

An application of the recurrence relation (4.38) to the estimate (4.39) yields

$$
\left\|\delta \boldsymbol{\phi}^{(\nu)}\right\|_{\infty} \leq \zeta_{2}\left\|\delta \boldsymbol{\phi}^{(\nu-1)}\right\|_{\infty}+\zeta_{3} \zeta_{1}^{\nu-3}\left\|\boldsymbol{\varphi}^{(1)}\right\|_{0, \infty, \Gamma}
$$

For either sufficiently small $\sigma_{\max }$ or sufficiently large $\rho_{\mathrm{PE}}$, we have $\zeta_{2}<1$. We have from the recurrence relation (4.40) that

$$
\begin{aligned}
\left\|\delta \boldsymbol{\phi}^{(\nu)}\right\|_{\infty} & \leq \zeta_{2}^{\nu-1}\left\|\boldsymbol{\phi}^{(1)}\right\|_{\infty}+\zeta_{3}\left(\zeta_{1}^{\nu-3}+\zeta_{2} \zeta_{1}^{\nu-4}+\cdots+\zeta_{2}^{\nu-4} \zeta_{1}+\zeta_{2}^{\nu-3}\right)\left\|\boldsymbol{\varphi}^{(1)}\right\|_{0, \infty, \Gamma} \\
& \leq \zeta_{2}^{\nu-1}\left\|\boldsymbol{\phi}^{(1)}\right\|_{\infty}+\zeta_{3}\left(\zeta_{1}+\zeta_{2}\right)^{\nu-3}\left\|\boldsymbol{\varphi}^{(1)}\right\|_{0, \infty, \Gamma} \rightarrow 0 \quad \text { as } \nu \rightarrow 0 .
\end{aligned}
$$

Here we assume that $\zeta_{1}+\zeta_{2}<1$ for sufficiently small $\sigma_{\max }$ and sufficiently large $\rho_{\mathrm{E}}$; or sufficiently large $\rho_{\mathrm{PE}}$ and $\rho_{\mathrm{E}}$, and have also used the fact that $\left\|\boldsymbol{\phi}^{(1)}\right\|_{\infty}<\infty$ and $\left\|\boldsymbol{\varphi}^{(1)}\right\|_{0, \infty, \Gamma}<\infty$ due to the well-posedness of the Foldy-Lax formulation (2.6) and the boundary integral equation (3.8).

Remark 4.3. Theorem 4.2 gives a sufficient condition in terms of the physical parameters. In particular if the separation distance among point scatterers and extended scatterers are sufficiently large, the physically motivated block Gauss-Seidel iteration is convergent. In practice, we find that the iteration still converges when separation distance among extended scatterers is much smaller than a wavelength, as long as the discretization of the boundary curves can resolve the separation distance, i.e., the discrete sampling can distinguish points on different obstacles, see numerical results in Section $\%$.

Remark 4.4. Though the discussion is focused on the two-dimensional Helmholtz equation, all the results on the well-posedness of the generalized Foldy-Lax formulation and the convergence of the block Gauss-Seidel iteration can be straightforwardly extended to the three-dimensional Helmholtz equation with the power changed to -1 for the separation distance parameters.

Remark 4.5. In addition to the block Gauss-Seidel iteration, the block Jacobi iteration may also be used to solve the generalized Foldy-Lax formulation (4.12)-(4.13). The block Jacobi iteration is equivalent to the series solution formulation, which is presented in [5] and proceeds as follows: Let $\boldsymbol{\phi}^{(0)}=(0, \ldots, 0)^{\top}$ and $\varphi_{k}^{(0)}(\mathbf{r})=0$ for $k=1, \ldots, n$, define $\phi^{(\nu)}$ and $\varphi_{k}^{(\nu)}$ for $\nu \geq 1$ by the solutions of the following system of equations

$$
A \phi^{(\nu)}=\phi_{\text {inc }}-\sum_{k=1}^{n} M_{k} \varphi_{k}^{(\nu-1)}
$$


and

$$
\left(\frac{1}{2} I+K_{k}^{\prime}-\mathrm{i} \eta S_{k}\right) \varphi_{k}^{(\nu)}=f_{k}-N_{k} \phi^{(\nu-1)}-\sum_{j=1}^{k-1}\left(K_{j}^{\prime}-\mathrm{i} \eta S_{j}\right) \varphi_{j}^{(\nu-1)}-\sum_{j=k+1}^{n}\left(K_{j}^{\prime}-\mathrm{i} \eta S_{j}\right) \varphi_{j}^{(\nu-1)}
$$

\section{Far-field pattern}

The far-field pattern of scattered wave plays a fundamental role in the inverse scattering theory since it contains important geometrical and physical information, e.g., location, shape, and the impedance of the boundary, of the scattering object. More specifically, given an incident field with incident direction $\mathbf{d}$, if $\psi$ is the scattered field, then $\psi$ has the asymptotic behavior

$$
\psi(\mathbf{r}, \mathbf{d})=\frac{e^{\mathrm{i} \kappa|\mathbf{r}|}}{\sqrt{|\mathbf{r}|}}\left[\psi_{\infty}(\hat{\mathbf{r}}, \mathbf{d})+O(|\mathbf{r}|)^{-1}\right] \quad \text { as }|\mathbf{r}| \rightarrow \infty
$$

uniformly in all directions $\hat{\mathbf{r}}=\mathbf{r} /|\mathbf{r}|$, where the function $\psi_{\infty}$ is called as the far-field pattern of the scattered field $\psi$, and $\hat{\mathbf{r}}=(\cos \beta, \sin \beta)$ is known as the observation direction and $\beta$ is the observation direction.

Recalling the following asymptotic behavior for the Hankel function (2.8) for large arguments and comparing with (5.1), we obtain from (2.5) and the following identity

$$
\left|\mathbf{r}-\mathbf{r}^{\prime}\right|=\sqrt{|\mathbf{r}|^{2}-2 \mathbf{r} \cdot \mathbf{r}^{\prime}+\left|\mathbf{r}^{\prime}\right|^{2}}=|\mathbf{r}|-\hat{\mathbf{r}} \cdot \mathbf{r}^{\prime}+O\left(|\mathbf{r}|^{-1}\right) \quad \text { as }|\mathbf{r}| \rightarrow \infty
$$

that the far-field pattern of the scattered field for the scattering problem of a set of $m$ point scatterers is

$$
\psi_{\infty, \mathrm{FL}}(\hat{\mathbf{r}}, \mathbf{d})=\frac{e^{\mathrm{i} \frac{\pi}{4}}}{\sqrt{8 \pi \kappa}} \sum_{j=1}^{m} \sigma_{j} \phi_{j}(\mathbf{d}) e^{-\mathrm{i} \kappa \hat{\mathbf{r}} \cdot \mathbf{r}_{j}},
$$

which is called the far-field pattern of the Foldy-Lax formulation. Under the Born approximation (2.10), the far-field pattern of the scattered field can be approximated by

$$
\psi_{\infty, \mathrm{B}}(\hat{\mathbf{r}}, \mathbf{d})=\frac{e^{\mathrm{i} \frac{\pi}{4}}}{\sqrt{8 \pi \kappa}} \sum_{j=1}^{m} \sigma_{j} e^{\mathrm{i} \kappa(\mathbf{d}-\hat{\mathbf{r}}) \cdot \mathbf{r}_{j}}
$$

which will be called the far-field pattern of the Born approximation.

It follows from the integral representation of the scattered field (3.7) and the asymptotic expansion of Green's function that the far-field pattern for the extended obstacle is given by

$$
\psi_{\infty, \mathrm{E}}(\hat{\mathbf{r}}, \mathbf{d})=-\frac{e^{\mathrm{i} \frac{\pi}{4}}}{\sqrt{8 \pi \kappa}} \int_{\Gamma} \partial_{\mathbf{n}^{\prime}} \phi\left(\mathbf{r}^{\prime} ; \mathbf{d}\right) e^{-\mathrm{i} \kappa \hat{\mathbf{r}} \cdot \mathbf{r}^{\prime}} d s\left(\mathbf{r}^{\prime}\right)
$$

Based on the integral equation representation (4.8) for the multiple scattering problem and the asymptotic behavior for Green's function, we may also obtain the far-field pattern of the scattered field for the generalized Foldy-Lax formulation

$$
\psi_{\infty, \mathrm{GFL}}(\hat{\mathbf{r}}, \mathbf{d})=\frac{e^{\mathrm{i} \frac{\pi}{4}}}{\sqrt{8 \pi \kappa}}\left[\sum_{j=1}^{m} \sigma_{j} \phi_{j}(\mathbf{d}) e^{-\mathrm{i} \kappa \hat{\mathbf{r}} \cdot \mathbf{r}_{j}}-\int_{\Gamma} \partial_{\mathbf{n}^{\prime}} \phi\left(\mathbf{r}^{\prime} ; \mathbf{d}\right) e^{-\mathrm{i} \kappa \hat{\mathbf{r}} \cdot \mathbf{r}^{\prime}} d s\left(\mathbf{r}^{\prime}\right)\right] .
$$


Remark 5.1. For the extended obstacle scatterer with more than one component, the far-field patterns for the extended obstacle and the generalized Foldy-Lax formulation can be written as

$$
\psi_{\infty, \mathrm{E}}(\hat{\mathbf{r}}, \mathbf{d})=-\frac{e^{\mathrm{i} \frac{\pi}{4}}}{\sqrt{8 \pi \kappa}} \sum_{k=1}^{n} \int_{\Gamma_{k}} \partial_{\mathbf{n}^{\prime}} \phi\left(\mathbf{r}^{\prime} ; \mathbf{d}\right) e^{-\mathrm{i} \kappa \hat{\mathbf{r}} \cdot \mathbf{r}^{\prime}} d s\left(\mathbf{r}^{\prime}\right) .
$$

and

$$
\psi_{\infty, \mathrm{GFL}}(\hat{\mathbf{r}}, \mathbf{d})=\frac{e^{\mathrm{i} \frac{\pi}{4}}}{\sqrt{8 \pi \kappa}}\left[\sum_{j=1}^{m} \sigma_{j} \phi_{j}(\mathbf{d}) e^{-\mathrm{i} \kappa \hat{\mathbf{r}} \cdot \mathbf{r}_{j}}-\sum_{k=1}^{n} \int_{\Gamma_{k}} \partial_{\mathbf{n}^{\prime}} \phi\left(\mathbf{r}^{\prime} ; \mathbf{d}\right) e^{-\mathrm{i} \kappa \hat{\mathbf{r}} \cdot \mathbf{r}^{\prime}} d s\left(\mathbf{r}^{\prime}\right)\right]
$$

Remark 5.2. The formulas of the far-field patterns can be extended to the three-dimensional Helmholtz equation with the constant changed to $1 / 4 \pi$ in front of the summation.

\section{$6 \quad$ Numerical techniques}

To compute the far-field patterns, we describe the numerical implementation of the Block GaussSeidel iterative method for the generalized Foldy-Lax formulation. As seen in (4.27) and (4.28), each step of the iteration essentially consists of solving two decoupled systems: a linear system of algebraic equations (2.4) for the point scatterers and a boundary integral equation (3.8) for a single extended obstacle scatterer. The linear system (2.4) can be easily solved by using a direct method such as the LU decomposition. In the following, we describe the Nyström method to solve the boundary integral equation (3.8). The method is similar to that in [2], where detailed discussion is given for the implementation of a boundary integral equation based on potential representations.

Recall the block Gauss-Seidel iteration (4.28) for the extended obstacle scatterers with more than one component, it is only required to solve a boundary integral equation for a single component at each step. To present the Nyström method, we may assume that the boundary curve $\Gamma$ possesses a regular analytic and $2 \pi$-periodic parametric representation of the form

$$
\mathbf{r}(t)=\left(x_{1}(t), x_{2}(t)\right), \quad 0 \leq t \leq 2 \pi
$$

in counterclockwise orientation satisfying $\left|\mathbf{r}^{\prime}(t)\right|=\left(\left|x_{1}^{\prime}(t)\right|^{2}+\left|x_{2}^{\prime}(t)\right|^{2}\right)^{1 / 2}>0$ for all $t$. Hence we assume that the obstacle has only one component. By straightforward calculations using $H_{0}^{(1)^{\prime}}=$ $-H_{1}^{(1)}$, we transform (3.8) into the parametric form

$$
\varphi(t)+\int_{0}^{2 \pi}[M(t, s)-\mathrm{i} \eta N(t, s)] \varphi(s) d s=f(t)
$$

where $\varphi(t):=\partial_{\mathbf{n}} \phi(\mathbf{r}(t)), f(t):=2\left(\partial_{\mathbf{n}}-\mathrm{i} \eta\right) \phi_{\text {inc }}(\mathbf{r}(t))$, and the integral kernels are given by

$$
\begin{aligned}
& M(t, s)=\frac{\mathrm{i} \kappa}{2} H_{1}^{(1)}\left(\kappa \rho_{1}(t, s)\right) \frac{\rho_{2}(t, s)}{\rho_{1}(t, s)} \frac{\left|\mathbf{r}^{\prime}(s)\right|}{\left|\mathbf{r}^{\prime}(t)\right|}, \\
& N(t, s)=\frac{\mathrm{i}}{2} H_{0}^{(1)}\left(\kappa \rho_{1}(t, s)\right)\left[\left|x_{1}^{\prime}(s)\right|^{2}+\left|x_{2}^{\prime}(s)\right|^{2}\right]^{1 / 2},
\end{aligned}
$$

for $t \neq s$. Here

$$
\begin{aligned}
& \rho_{1}(t, s)=\left(\left|x_{1}(t)-x_{1}(s)\right|^{2}+\left|x_{2}(t)-x_{2}(s)\right|^{2}\right)^{1 / 2} \\
& \rho_{2}(t, s)=x_{2}^{\prime}(t)\left(x_{1}(s)-x_{1}(t)\right)-x_{1}^{\prime}(t)\left(x_{2}(s)-x_{2}(t)\right) .
\end{aligned}
$$


It follows from the expansion of the Hankel function that the kernels $M$ and $N$ have logarithmic singularities at $t=s$. Hence, following [2], we split the kernels into

$$
\begin{aligned}
& M(t, s)=M_{1}(t, s) \ln \left(4 \sin ^{2} \frac{t-s}{2}\right)+M_{2}(t, s), \\
& N(t, s)=N_{1}(t, s) \ln \left(4 \sin ^{2} \frac{t-s}{2}\right)+N_{2}(t, s),
\end{aligned}
$$

where

$$
\begin{aligned}
& M_{1}(t, s)=-\frac{\kappa}{2 \pi} J_{1}\left(\kappa \rho_{1}(t, s)\right) \frac{\rho_{2}(t, s)}{\rho_{1}(t, s)} \frac{\left|\mathbf{r}^{\prime}(s)\right|}{\left|\mathbf{r}^{\prime}(t)\right|} \\
& M_{2}(t, s)=M(t, s)-M_{1}(t, s) \ln \left(4 \sin ^{2} \frac{t-s}{2}\right) \\
& N_{1}(t, s)=-\frac{1}{2 \pi} J_{0}\left(\kappa \rho_{1}(t, s)\right)\left[\left|x_{1}^{\prime}(s)\right|^{2}+\left|x_{2}^{\prime}(s)\right|^{2}\right]^{1 / 2} \\
& N_{2}(t, s)=N(t, s)-N_{1}(t, s) \ln \left(4 \sin ^{2} \frac{t-s}{2}\right) .
\end{aligned}
$$

The kernels $M_{1}, M_{2}, N_{1}$, and $N_{2}$ turn out to be analytic. In particular, using the expansions of the Bessel functions, we can deduce the diagonal terms

$$
M_{1}(t, t)=0, \quad M_{2}(t, t)=\frac{1}{2 \pi} \frac{x_{2}^{\prime}(t) x_{1}^{\prime \prime}(t)-x_{1}^{\prime}(t) x_{2}^{\prime \prime}(t)}{\left|x_{1}^{\prime}(t)\right|^{2}+\left|x_{2}^{\prime}(t)\right|^{2}},
$$

and

$$
N_{2}(t, t)=\left[\frac{\mathrm{i}}{2}-\frac{E}{\pi}-\frac{1}{2 \pi} \ln \left(\frac{\kappa^{2}}{4}\left|\mathbf{r}^{\prime}(t)\right|^{2}\right)\right]\left|\mathbf{r}^{\prime}(t)\right|,
$$

where $E$ is Euler's constant. We note that despite the continuity of the kernel $M$, for numerical accuracy it is advantageous to separate the logarithmic part of $M$ since the derivatives of $M$ fail to be continuous at $t=s$.

Hence, we have to numerically solve an integral of the form

$$
\varphi(t)+\int_{0}^{2 \pi} K(t, s) \varphi(s) d s=f(t), \quad 0 \leq t \leq 2 \pi
$$

where the kernel can be written in the form

$$
K(t, s)=K_{1}(t, s) \ln \left(4 \sin ^{2} \frac{t-s}{2}\right)+K_{2}(t, s)
$$

Here

$$
\begin{aligned}
& K_{1}(t, s)=M_{1}(t, s)-\mathrm{i} \eta N_{1}(t, s), \\
& K_{2}(t, s)=M_{2}(t, s)-\mathrm{i} \eta N_{2}(t, s) .
\end{aligned}
$$

The Nyström method consists in the straightforward approximation of the integrals by quadrature formulas. In our case, for the $2 \pi$-periodic integrands, we choose an equidistant set of knots $t_{j}:=$ $j \pi / n, j=0, \ldots, 2 n-1$, and use the quadrature rule

$$
\int_{0}^{2 \pi} \ln \left(4 \sin ^{2} \frac{t-s}{2}\right) g(s) d s \approx \sum_{j=0}^{2 n-1} R_{j}^{(n)}(t) g\left(t_{j}\right), \quad 0 \leq t \leq 2 \pi
$$


with the quadrature weights given by

$$
R_{j}^{(n)}(t):=-\frac{2 \pi}{n} \sum_{m=1}^{n-1} \frac{1}{m} \cos m\left(t-t_{j}\right)-\frac{\pi}{n^{2}} \cos n\left(t-t_{j}\right), \quad j=0, \ldots, 2 n-1,
$$

and the trapezoidal rule

$$
\int_{0}^{2 \pi} g(t) d t \approx \frac{\pi}{n} \sum_{j=1}^{2 n-1} g\left(t_{j}\right)
$$

In the Nyström method, the integral equation (6.2) is replaced by the approximation equation after applying the (6.3) and (6.4)

$$
\varphi^{(n)}(t)+\sum_{j=0}^{2 n-1}\left[R_{j}^{(n)}(t) K_{1}\left(t, t_{j}\right)+\frac{\pi}{n} K_{2}\left(t, t_{j}\right)\right] \varphi^{(n)}\left(t_{j}\right)=f(t)
$$

for $0 \leq t \leq 2 \pi$. In particular, for any solution of $(6.5)$ the value $\varphi_{i}^{(n)}=\varphi^{(n)}\left(t_{i}\right), i=0, \ldots, 2 n-1$, at the quadrature points trivially satisfy the linear system

$$
\varphi_{i}^{(n)}+\sum_{j=0}^{2 n-1}\left[R_{|i-j|}^{(n)} K_{1}\left(t_{i}, t_{j}\right)+\frac{\pi}{n} K_{2}\left(t_{i}, t_{j}\right)\right] \varphi_{j}^{(n)}=f\left(t_{i}\right)
$$

for $i=0, \ldots, 2 n-1$, where

$$
R_{j}^{(0)}:=R_{j}^{(n)}(0)=-\frac{2 \pi}{n} \sum_{m=1}^{n-1} \frac{1}{m} \cos \frac{j m \pi}{n}-\frac{(-1)^{j} \pi}{n^{2}}, \quad j=0, \ldots, 2 n-1 .
$$

To demonstrate the performance of the above method for solving the boundary integral equation (3.8), we take the benchmark example in [2] and consider the scattering of a plane wave by a kiteshaped obstacle, which is described by the parametric representation

$$
\mathbf{r}(t)=(\cos t+0.65 \cos 2 t-0.65,1.5 \sin t), \quad 0 \leq t \leq 2 \pi,
$$

as seen in the middle of Figure 2. The far-field pattern of the scattered wave for the obstacle scattering problem is given by (5.4), which can be evaluated again by the trapezoidal rule after solving the integral equation for $\partial_{\mathbf{n}} \phi$. Table 1 gives numerical approximations for the far-field pattern $\psi_{\infty, \mathrm{E}}(\hat{\mathbf{r}}, \mathbf{d})$ in the forward direction $\hat{\mathbf{r}}=\mathbf{d}$ and the backward direction $\hat{\mathbf{r}}=-\mathbf{d}$. The direction $\mathbf{d}$ of the incident wave is $\mathbf{d}=(1,0)$ and the coupling parameter $\eta=\kappa$. Note that the exponential convergence is clearly exhibited.

\section{$7 \quad$ Numerical experiments}

In this section, we present some numerical examples for the computation of the far-field patterns for the incident angle $\alpha \in[0,2 \pi]$ and the observation angle $\beta \in[0,2 \pi]$. As comparisons, we will show the different far-field patterns: the far-field pattern via the Born approximation $\psi_{\infty, \mathrm{B}}$ for a group of point scatterers, the far-field pattern via the original Foldy-Lax formulation $\psi_{\infty, \mathrm{FL}}$ for a group of point scatterers, the far-field pattern via the boundary integral equation for extended obstacle scatterers $\psi_{\infty, \mathrm{E}}$, and the far-field pattern via the generalized Foldy-Lax formulation for both point and extended obstacle scatterers. In Example 1 and Example 2, the wavenumber $\kappa$ is 
Table 1: Numerical results of the Nyström's method to solve the boundary integral equation for a kite-shaped obstacle.

\begin{tabular}{crrrrr}
\hline & $n$ & $\operatorname{Re} \psi_{\infty, \mathrm{E}}(\mathbf{d}, \mathbf{d})$ & $\operatorname{Im} \psi_{\infty, \mathrm{E}}(\mathbf{d}, \mathbf{d})$ & $\operatorname{Re} \psi_{\infty, \mathrm{E}}(-\mathbf{d}, \mathbf{d})$ & $\operatorname{Im} \psi_{\infty, \mathrm{E}}(-\mathbf{d}, \mathbf{d})$ \\
\hline \hline \multirow{4}{*}{$\kappa=1$} & -1.62399616 & 0.60308809 & 1.39015276 & 0.09425143 \\
& 16 & -1.62746853 & 0.60222418 & 1.39696605 & 0.09499467 \\
& 32 & -1.62745750 & 0.60222603 & 1.39694483 & 0.09499650 \\
& 64 & -1.62745750 & 0.60222603 & 1.39694483 & 0.09499650 \\
\hline \multirow{2}{*}{$\kappa=5$} & 16 & -2.47821459 & 1.72919957 & -0.39350495 & 0.11897067 \\
& 32 & -2.47554472 & 1.68747934 & -0.19945835 & 0.06015728 \\
& 64 & -2.47554477 & 1.68747932 & -0.19945836 & 0.06015726 \\
\hline
\end{tabular}

taken to be $4 \pi$, i.e., the wavelength is taken to be $\lambda=2 \pi / \kappa=0.5$. In Example 3 , the wavenumber $\kappa$ is taken to be $2 \pi$ which accounts for the wavelength $\lambda=2 \pi / \kappa=1.0$.

Example 1. Consider a group of twenty equally distributed point scatterers on a circle with radius 3 and a kite-shaped extended obstacle with the parametric representation given by

$$
\mathbf{r}(t)=(\cos t+0.65 \cos 2 t-0.65,1.5 \sin t), \quad 0 \leq t \leq 2 \pi
$$

as seen in Figure 2. The scattering coefficient $\sigma_{j}$ is the same for all the twenty point scatterers, which is $\sigma_{j}=\sigma=1.0$ for Figures 3-7. Figures 3 and 4 show the far-field patterns for the point scatterers, as seen in the left of Figure 2. Figure 3 shows the real and imaginary parts of the far-field pattern for the scattered field computed from the Born approximation (2.10), where the multiple scattering is neglected among the point scatterers. As a comparison, Figure 4 shows the real and imaginary parts of the far-field pattern for the scattered field computed from the Foldy-Lax formulation (2.5), where the multiple scattering is taken into account among the point scatterers. As we can see from Figure 3 and Figure 4, the difference is clear between the far-field pattern $\psi_{\infty, \mathrm{B}}$ and the far-field pattern $\psi_{\infty, \mathrm{FL}}$. Figure 5 shows the far-field pattern for the single kite-shaped extended obstacle scatterer, as seen in the middle of Figure 2. It shows the real and imaginary parts of the far-field pattern for the scattered field (3.7) computed from the boundary integral equation (3.8). Figure 6 shows the far-field pattern for the heterogeneous medium with both point and extended obstacle scatterers, as seen in the right of Figure 2. It shows the real and imaginary parts of the far-field pattern for the scattered field (4.8) computed from the generalized Foldy-Lax formulation (4.10) and (4.11). Comparing with the far-field pattern in Figure 5, the far-field pattern in Figure 6 displays a lot of small oscillation, which comes from the presence of the point scatterers. In order to show whether the generalized Foldy-Lax formulation captures the multiple scattering between the point scatterers and the extended kite-shaped obstacle scatterer, Figure 7 shows only the portion of the far-field pattern due to the multiple scattering by subtracting $\psi_{\infty, \mathrm{E}}$ and $\psi_{\infty, \mathrm{FL}}$ from $\psi_{\infty, \mathrm{GFL}}$, i.e., the far-field pattern $\psi_{\infty, \mathrm{GFL}}-\psi_{\infty, \mathrm{E}}-\psi_{\infty, \mathrm{FL}}$.

To show how the convergence of the Block Gauss-Seidel iteration and the block Jacobi iteration depend on the point scatterers, such as the scattering coefficients and the number of point scatterers, 

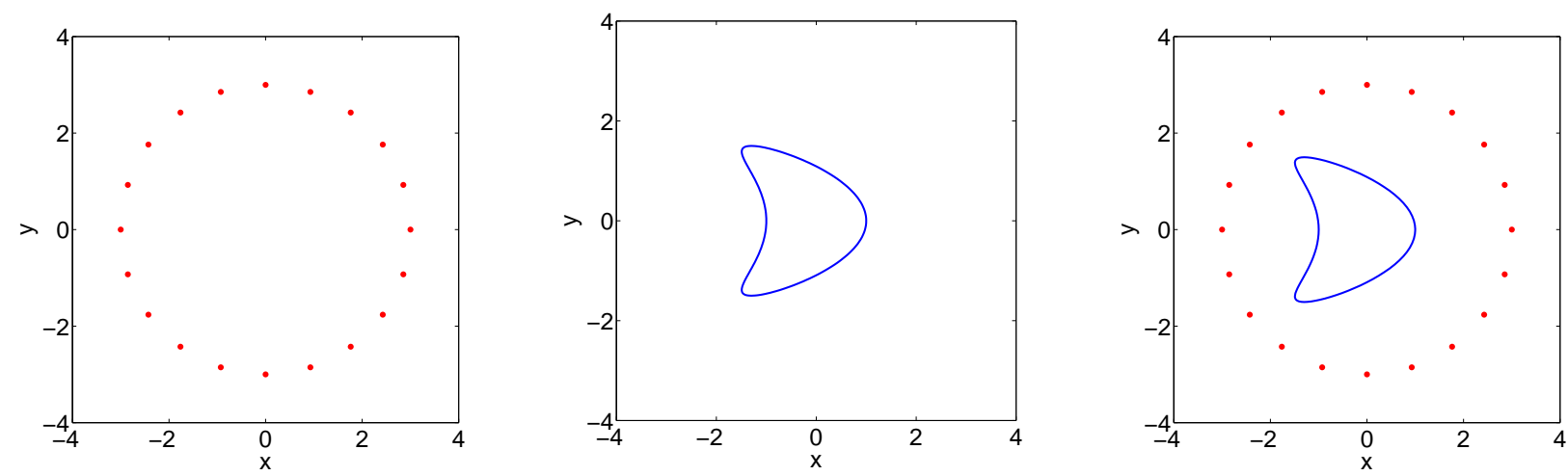

Figure 2: Uniformly distributed point scatterers and a kite-shaped obstacle scatterer in Example 1. (left) a set of twenty equally spaced point scatterers on a circle with radius 3; (middle) a kiteshaped extended obstacle scatterer; (right) mixed scatterers of a kite-shaped obstacle surrounded by a set of twenty equally spaced point scatterers.

we define the error between two consecutive approximations

$$
e_{\mathrm{FL}}=\left\|\phi^{(\nu)}-\phi^{(\nu-1)}\right\|_{2} \quad \text { and } \quad e_{\mathrm{E}}=\left\|\varphi^{(\nu)}-\varphi^{(\nu-1)}\right\|_{L^{2}(\Gamma)},
$$

where $\nu$ is the number of iteration. Define

$$
e_{\mathrm{GFL}}=\max \left\{e_{\mathrm{FL}}, e_{\mathrm{E}}\right\} .
$$

Given the twenty equally distributed point scatterers with equal scattering coefficient on the circle with radius 3 , Figure 8 shows the error $e_{\mathrm{GFL}}$ of two consecutive approximations using $\sigma_{j}=\sigma=$ $1,2,4,8$. As can be seen from Figure 8, the larger of the scattering coefficient is, the stronger the multiple scattering among the point scatterers, and thus more iterations are needed to reach the same level of accuracy. Given the scattering coefficients $\sigma_{j}=\sigma=1.0$, Figure 9 investigates the error $e_{\mathrm{GFL}}$ of two consecutive approximations using different number of equally spaced point scatterers $m=20,100,200,300$ on the circle with radius 3. Similarly, it can be observed that more iterations are needed to reach the same level of accuracy for more number of point scatterers. As expected, it can be seen from Figure 8 and Figure 9 that the block Gauss-Seidel iteration displays a faster convergence than the block Jacobi iteration.

Example 2. Consider a group of one hundred randomly distributed point scatterers in annulus with radii bounded blow by 3 and above by 4 and two extended obstacle scatterers: one is a circular extended scatterer with the parametric representation given by

$$
\mathbf{r}_{1}(t)=(\cos t-1.5, \sin t), \quad 0 \leq t \leq 2 \pi ;
$$

and the other one is a kite-shaped extended scatterer with the parametric representation given by

$$
\mathbf{r}_{2}(t)=(\cos t+0.65 \cos 2 t+0.85,1.5 \sin t), \quad 0 \leq t \leq 2 \pi,
$$

as seen in Figure 10. The scattering coefficient $\sigma_{j}$ is taken as a random number from the interval $(0,1)$ for $j=1, \ldots, 100$ in Example 2. Figures 11 and 12 concern with the far-field patterns for the randomly distributed point scatterers, as seen in the left of Figure 10. Figure 11 shows the real and imaginary parts of the far-field pattern for the scattered field computed from the Born approximation (2.10). As a comparison, Figure 12 shows the real and imaginary parts of the far-field pattern for the scattered field computed from the Foldy-Lax formulation (2.5), where 

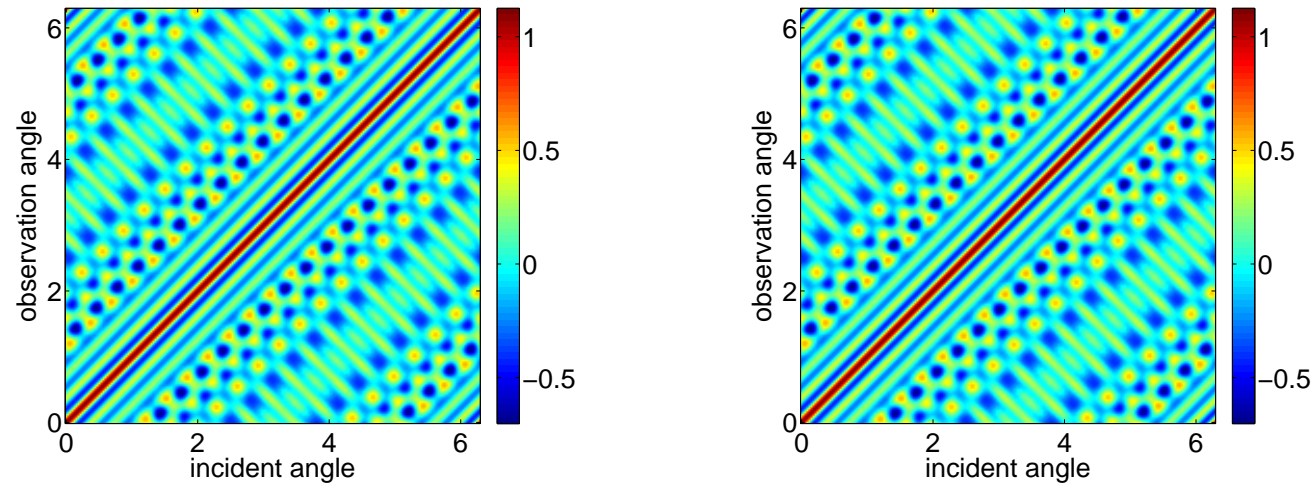

Figure 3: The far-field pattern $\psi_{\infty, \mathrm{B}}$ of the scattered field from the uniformly distributed point scatterers in Example 1 via the Born approximation. (left) real part; (right) imaginary part.
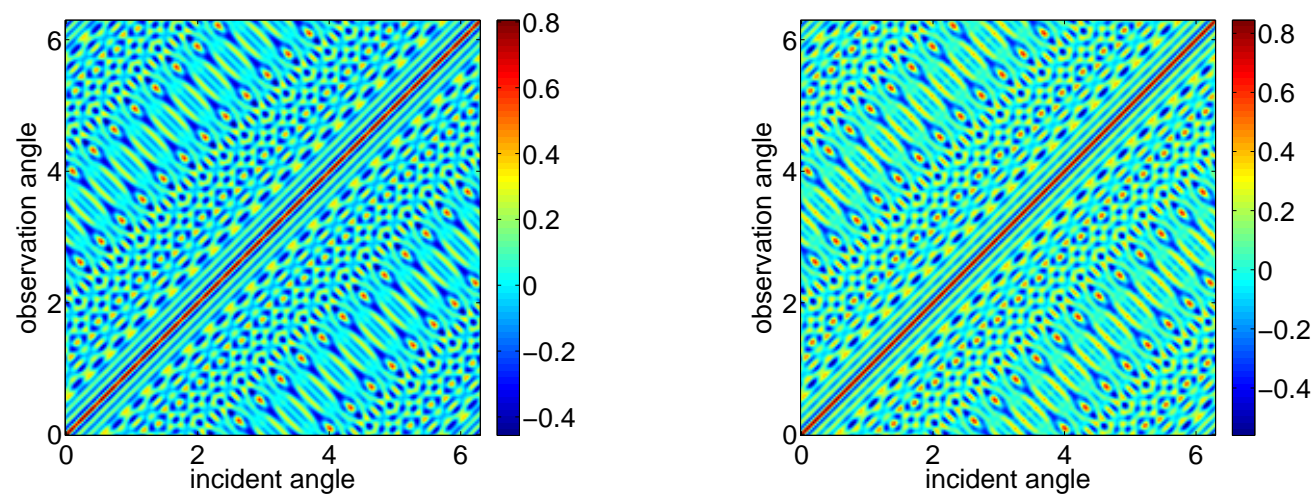

Figure 4: The far-field pattern $\psi_{\infty, \mathrm{FL}}$ of the scattered field from the uniformly distributed point scatterers in Example 1 via the Foldy-Lax formulation. (left) real part; (right) imaginary part.
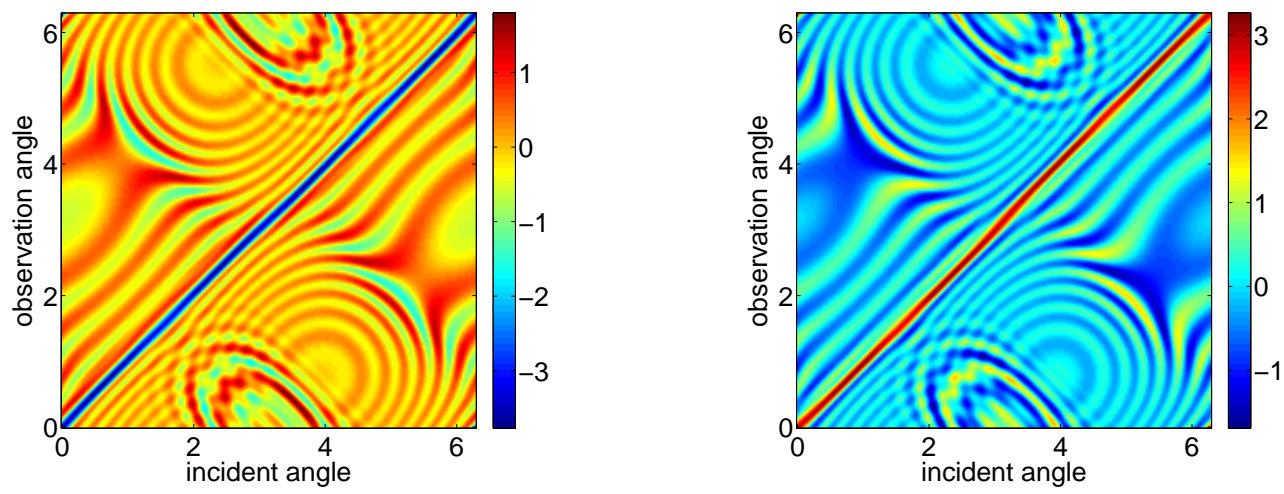

Figure 5: The far-field pattern $\psi_{\infty, \mathrm{E}}$ of the scattered field from the kite-shaped extended obstacle scatterer in Example 1 via the boundary integral equation. (left) real part; (right) imaginary part. 

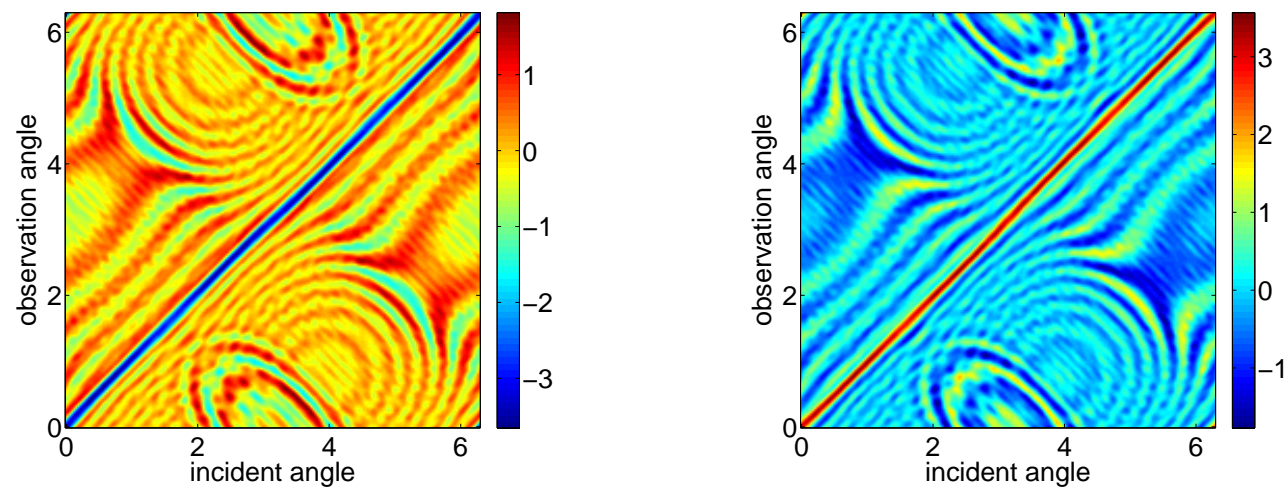

Figure 6: The far-field pattern $\psi_{\infty, \mathrm{GFL}}$ of the scattered field from the uniformly distributed point scatterers and the kite-shaped extended obstacle scatterer in Example 1 via the generalized FoldyLax formulation. (left) real part; (right) imaginary part.
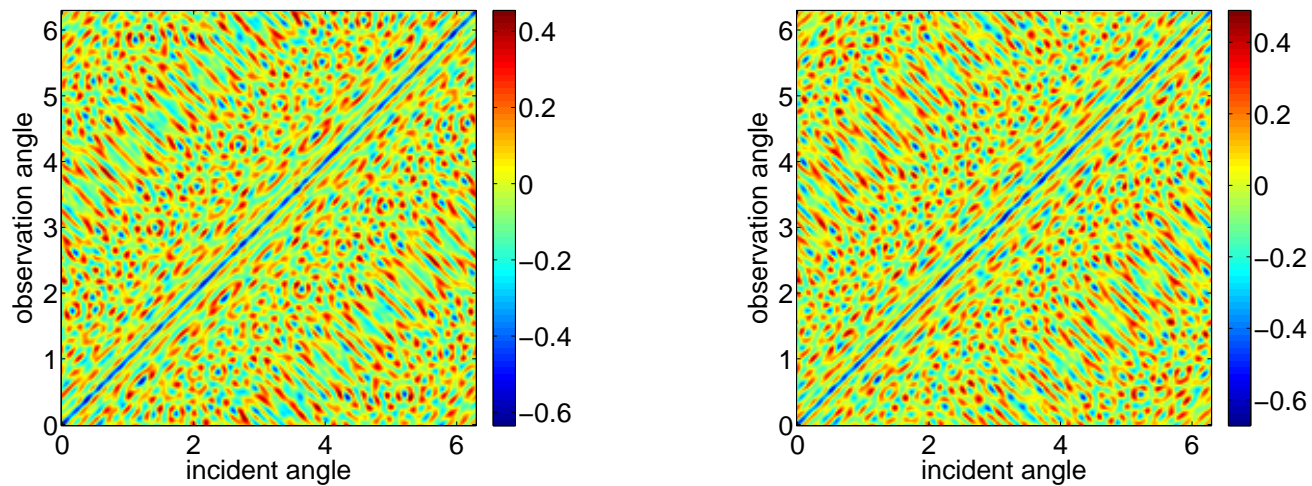

Figure 7: The difference of the far-field patterns $\psi_{\infty, \mathrm{GFL}}-\psi_{\infty, \mathrm{E}}-\psi_{\infty, \mathrm{FL}}$ from the uniformly distributed point scatterers and the kite-shaped extended obstacle scatterer in Example 1. (left) real part; (right) imaginary part.
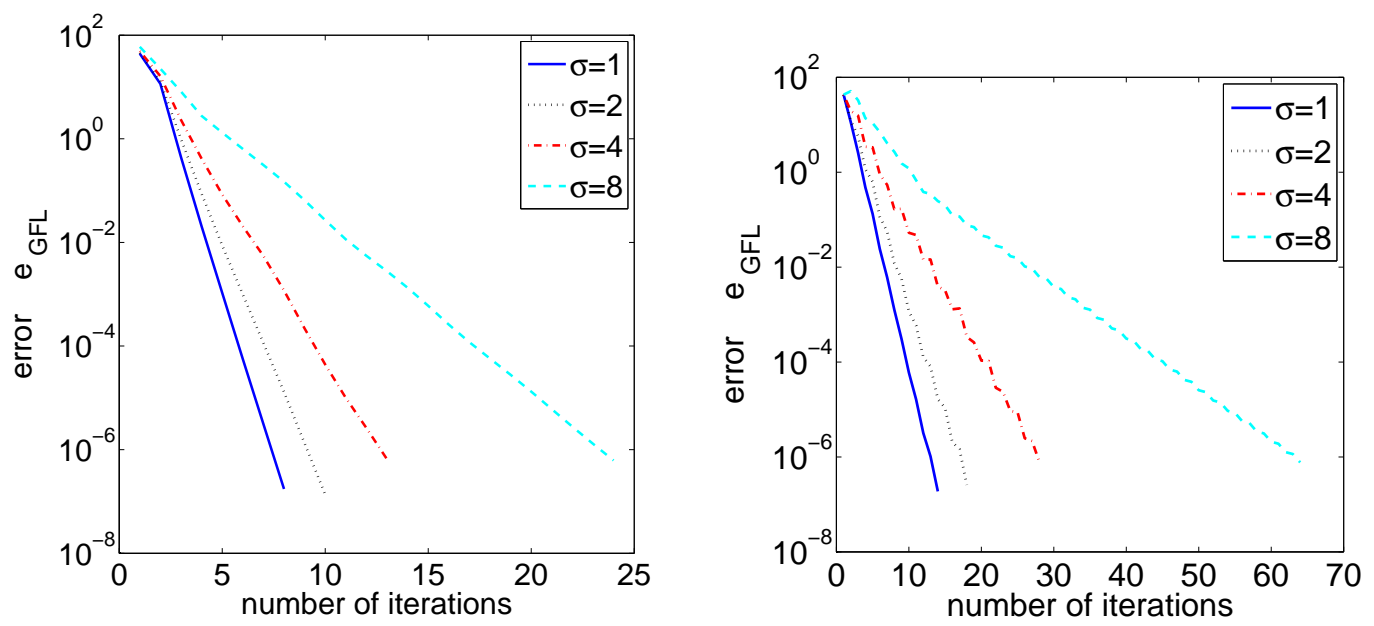

Figure 8: The error of two consecutive approximations is given against the number of iterations with different scattering coefficients for Example 1. (left) block Gauss-Seidel iteration; (right) block Jacobi iteration. 

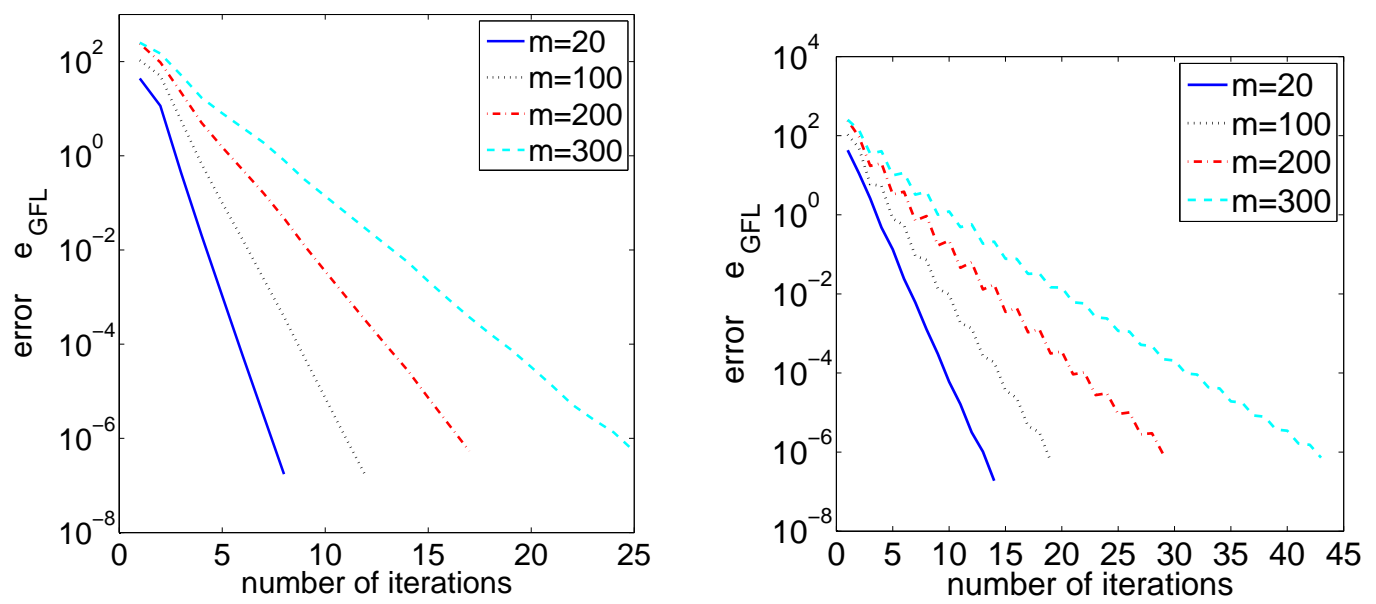

Figure 9: The error of two consecutive approximations is given against the number of iterations with different number of point scatterers for Example 1. (left) block Gauss-Seidel iteration; (right) block Jacobi iteration.

the multiple scattering is taken into account among the point scatterers. Due to the randomly distributed point scatterers, Figures 11 and 12 show no symmetric patterns, which are different from Figure 3 and 4 for the equally distributed point scatterers. Figure 13 shows the far-field pattern for the two extended obstacle scatterers, as seen in the middle of Figure 10. It shows the real and imaginary parts of the far-field pattern for the scattered field (3.7) computed from the boundary integral equation (3.8). Figure 14 shows the far-field pattern for the heterogeneous medium with both point and extended obstacle scatterers, as seen on the right of Figure 10. It shows the real and imaginary parts of the far-field pattern for the scattered field (4.8) computed from the generalized Foldy-Lax formulation (4.10) and (4.11). Again, in order to show whether the generalized Foldy-Lax formulation captures the multiple scattering between the point scatterers and the extended kite-shaped obstacle scatterer, Figure 15 shows only the portion of the far-field pattern due to the multiple scattering by subtracting $\psi_{\infty, \mathrm{E}}$ and $\psi_{\infty, \mathrm{FL}}$ from $\psi_{\infty, \mathrm{GFL}}$, i.e., the far-field pattern $\psi_{\infty, \mathrm{GFL}}-\psi_{\infty, \mathrm{E}}-\psi_{\infty, \mathrm{FL}}$.

Given the one hundred randomly distributed point scatterers with scattering coefficient chosen as a random number from the interval $(0,1)$, Figure 16 plots the error $e_{\mathrm{GFL}}$ of two consecutive approximations with different separation distance of the two extended scatterers, $\rho_{\mathrm{E}}=$ $0.25 \lambda, 0.5 \lambda, 1.0 \lambda, 2.0 \lambda$, in order to show how the convergence of the Block Gauss-Seidel iteration and the block Jacobi iteration depend on the separation distance between the extended scatterers $\rho_{\mathrm{E}}$. Theorem 4.2 gives a sufficient condition for the convergence of the physically motivated GaussSeidel iteration, which requires that the separation distance among scatterers to be sufficiently large. In practice, the iteration still converges when the separation distance $\rho_{\mathrm{E}}$ of the two extended scatterers is as close as $0.25 \lambda$ as long as the discretization of the boundary can resolve the separation distance. Surprisingly, the number of iterations is not necessary to increase as the separation distance between the two extended scatterer $\rho_{\mathrm{E}}$ decreases for both the block Gauss-Seidel iteration and the block Jacobi iteration. The number of iterations increases as the separation distance $\rho_{\mathrm{E}}$ decreases to $2 \lambda$; and then it decreases as the separation distance further decreases to $0.25 \lambda$, as seen in Figure 16. As usual, the block Gauss-Seidel iteration exhibits a faster convergence than the block Jacobi iteration.

Example 3. We show an example to illustrate that the developed generalized Foldy-Lax formulation and the proposed block Gauss-Seidel method can be extended to the three-dimensional 

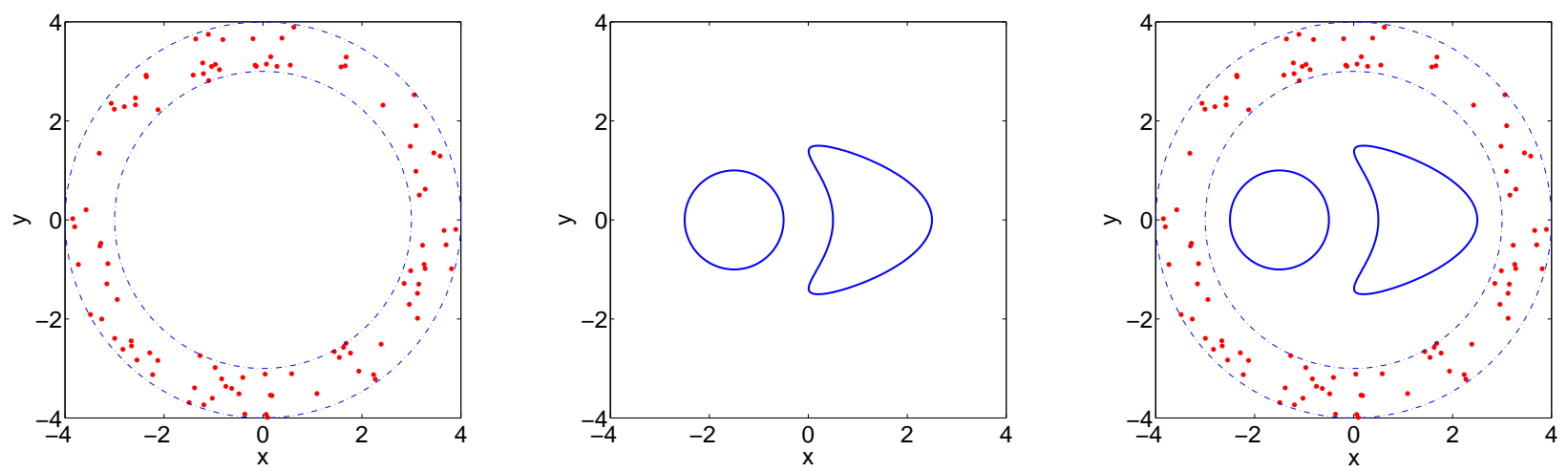

Figure 10: Randomly distributed point scatterers and two obstacle scatterers in Example 2. (left) a group of one hundred of randomly distributed point scatterers in the annulus with radii bounded blow by 3 and above by 4; (middle) a kite-shaped and a circle-shaped extended obstacle scatterers; (right) mixed scatterers of a kite-shaped and a circle-shaped obstacle scatterers surrounded by a set of one hundred randomly distributed point scatterers.
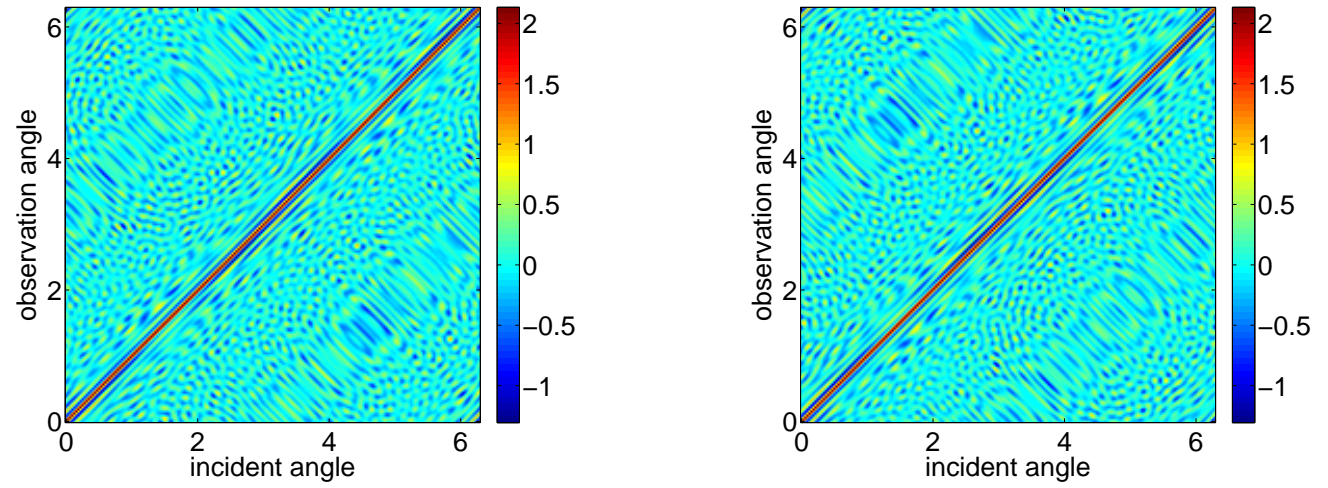

Figure 11: The far-field pattern $\psi_{\infty, \mathrm{B}}$ of the scattered field from the uniformly distributed point scatterers in Example 2 via the Born approximation. (left) real part; (right) imaginary part.
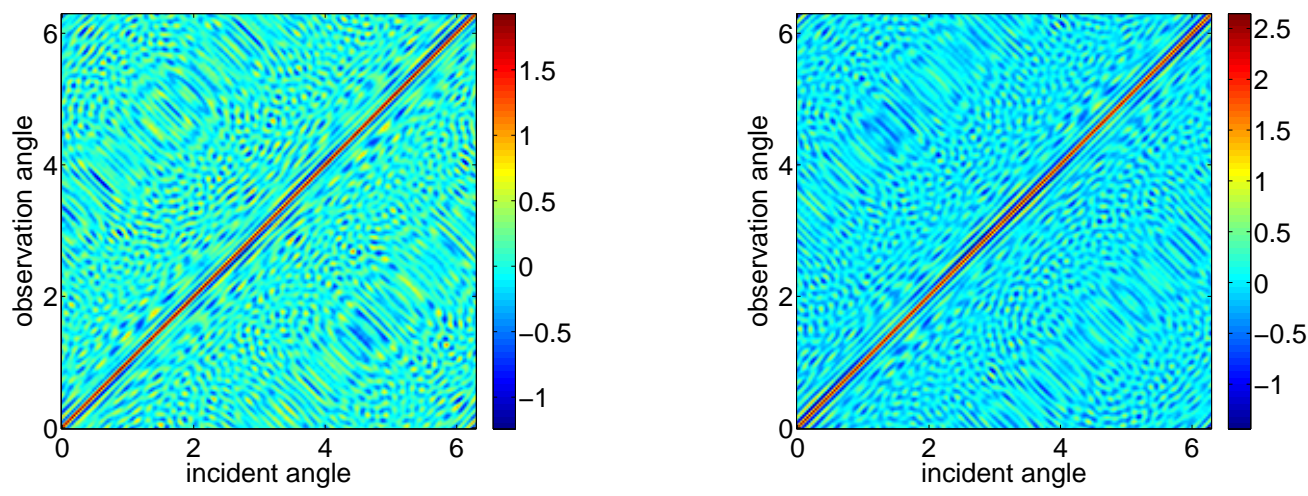

Figure 12: The far-field pattern $\psi_{\infty, \mathrm{FL}}$ of the scattered field from the uniformly distributed point scatterers in Example 2 via the Foldy-Lax formulation. (left) real part; (right) imaginary part. 

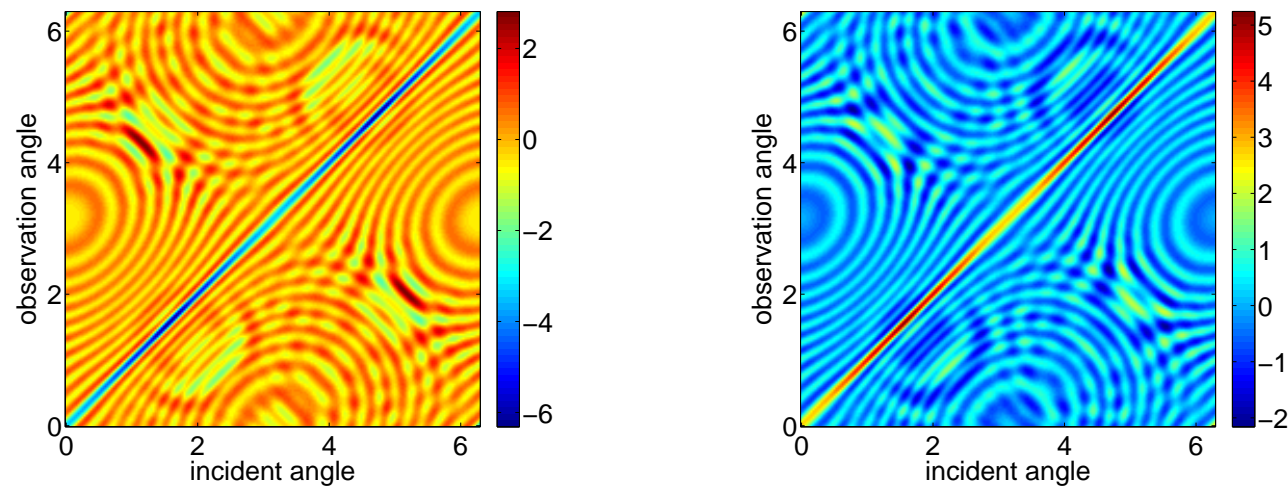

Figure 13: The far-field pattern $\psi_{\infty, \mathrm{E}}$ of the scattered field from the kite-shaped extended obstacle scatterer in Example 2 via the boundary integral equation. (left) real part; (right) imaginary part.
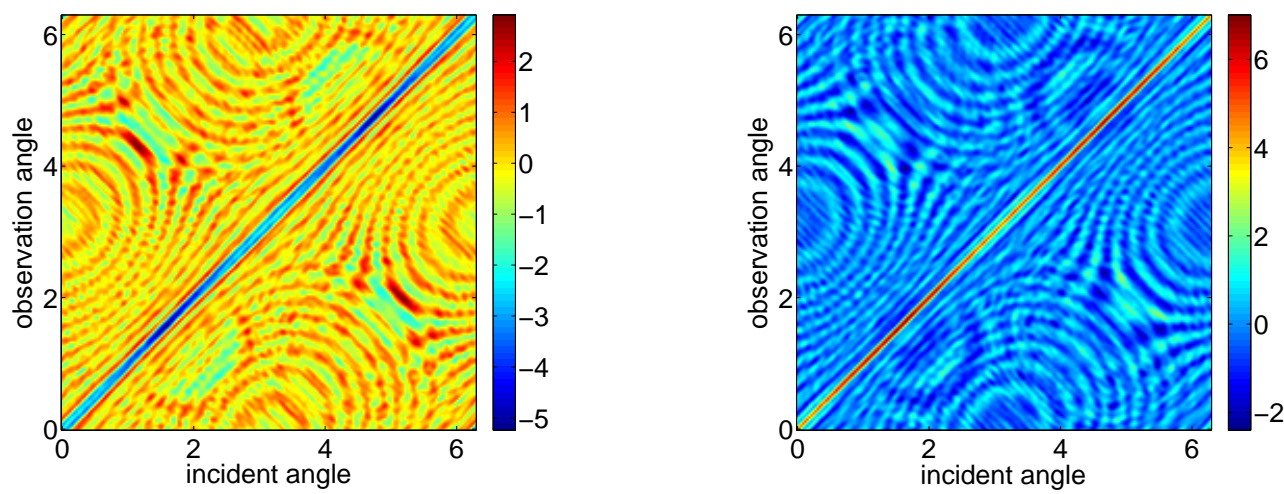

Figure 14: The far-field pattern $\psi_{\infty, \mathrm{GFL}}$ of the scattered field from the uniformly distributed point scatterers and the kite-shaped extended obstacle scatterer in Example 2 via the generalized FoldyLax formulation. (left) real part; (right) imaginary part.
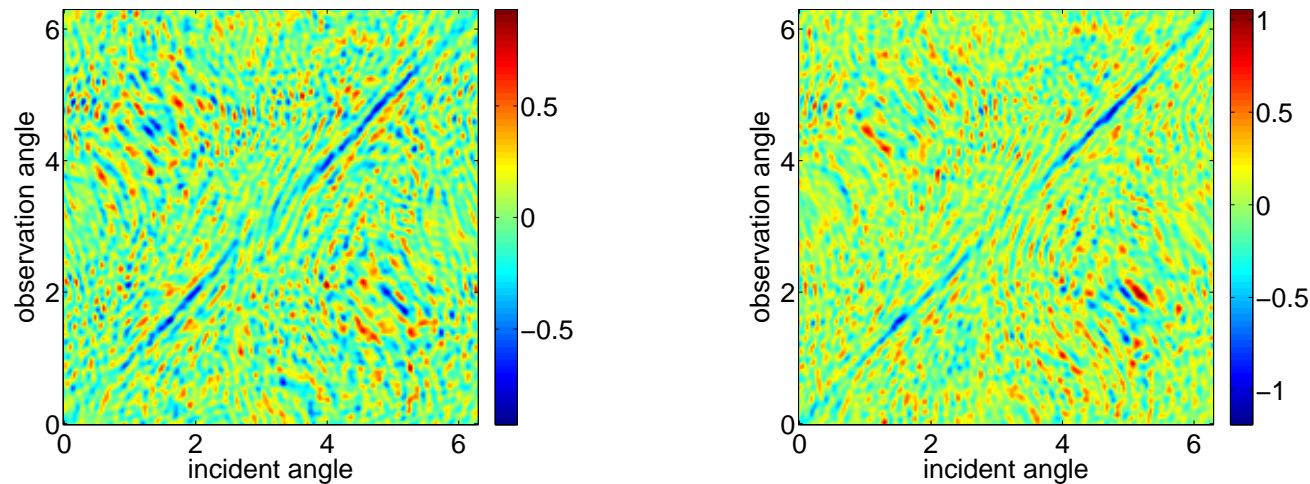

Figure 15: The difference of the far-field patterns $\psi_{\infty, \mathrm{GFL}}-\psi_{\infty, \mathrm{E}}-\psi_{\infty, \mathrm{FL}}$ from the uniformly distributed point scatterers and the kite-shaped extended obstacle scatterer in Example 2. (left) real part; (right) imaginary part. 

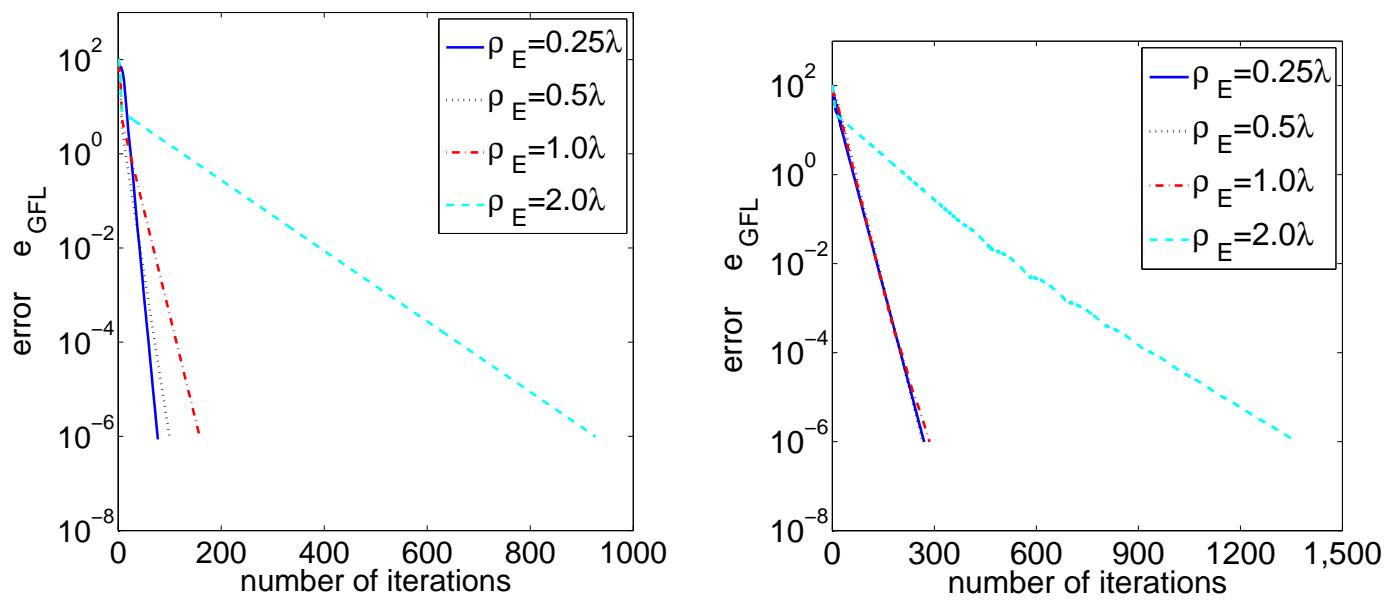

Figure 16: Error of two consecutive approximations using different separation distance for Example 2. (left) block Gauss-Seidel iteration; (right) block Jacobi iteration.

Helmholtz equation, with the power of the separation distance changed to -1 and the constant in the far-field pattern changed to $1 / 4 \pi$. We choose the Galerkin boundary element method for solving the three-dimensional boundary integral equations, and refer to [5] for detailed descriptions of the method. In this example, we consider a group of twenty equally distributed point scatterers on a circle with radius 2 on the $x y$-plane, as seen in left of Figure 17. The scattering coefficients $\sigma_{j}$ is chosen to be all equal, i.e., $\sigma_{j}=\sigma=1.0$. The centers of two spherical obstacle scatterers with the same radius of 0.5 are located at $(-1.0,0.0,0.0)$ and $(1.0,0.0,0.0)$, respectively, as seen in the middle of Figure 17. The far-field patterns are plotted on the unit sphere as a function of the variables for the latitudinal angle and the longitudinal angle varying from 0 to $\pi$ and from 0 to $2 \pi$, respectively. All the figures are shown from the incident direction of $\mathbf{d}=(0,0,1)$. Figure 18 shows the real and imaginary parts of the far-field pattern, $\psi_{\infty, \mathrm{FL}}$, for the scattered field computed from the original Foldy-Lax formulation. Figure 19 shows the far-field pattern, $\psi_{\infty, \mathrm{E}}$, for the two spherical scatterers by using the boundary integral equation, and Figure 20 plots the far-field pattern, $\psi_{\infty, \mathrm{GFL}}$, for the mixed scatterers by using the generalized Foldy-Lax formulation. Finally, Figure 21 shows the portion of the far-field pattern arising from the multiple scattering among all scatterers by subtracting $\psi_{\infty, \mathrm{E}}$ and $\psi_{\infty, \mathrm{FL}}$ from $\psi_{\infty, \mathrm{GFL}}$, i.e., the far-field pattern $\psi_{\infty, \mathrm{GFL}}-\psi_{\infty, \mathrm{E}}-\psi_{\infty, \mathrm{FL}}$. Figure 22 plots the error $e_{\mathrm{GFL}}$ of two consecutive approximations with different separation distance of the two spheres, $\rho_{\mathrm{E}}=0.25 \lambda, 0.5 \lambda, 1.0 \lambda$ to show how the convergence of the Block Gauss-Seidel iteration and the block Jacobi iteration depend on the separation distance between the extended scatterers for the three-dimensional Helmholtz equation. Remarkably, the number of iterations and convergence is almost independent of the separation distance in 3D for both methods of iteration; while the block Gauss-Seidel iteration shows a faster convergence than the block Jacobi iteration.

\section{Conclusions}

We presented an efficient algorithm for the generalized Foldy-Lax formulation to capture multiple scattering among a group of isotropic point scatterers and extended obstacle scatterers. The improved formulation is based on a coupled system which combines the original Foldy-Lax formulation and a uniquely solvable boundary integral equation for exterior boundary value problem. The uniqueness and existence of the solution for the improved formulation were given in terms of the 

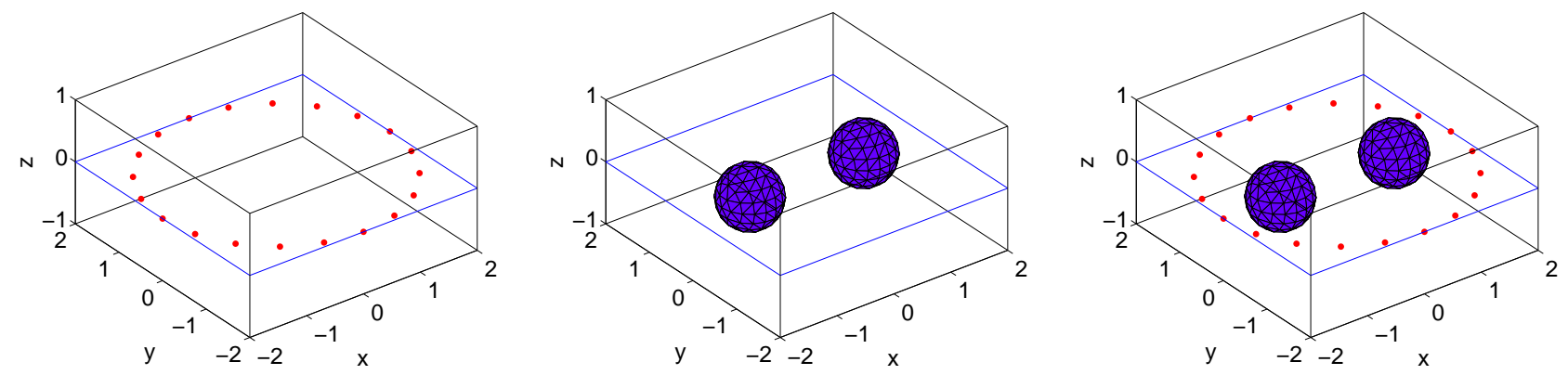

Figure 17: Uniformly distributed point scatterers and two spherical obstacle scatterer in Example 3. (left) a set of twenty equally spaced point scatterers on a circle with radius 2 on the $x y$ plane; (middle) two spherical extended obstacle scatterers; (right) mixed scatterers of two spherical obstacle surrounded by a set of twenty equally spaced point scatterers.
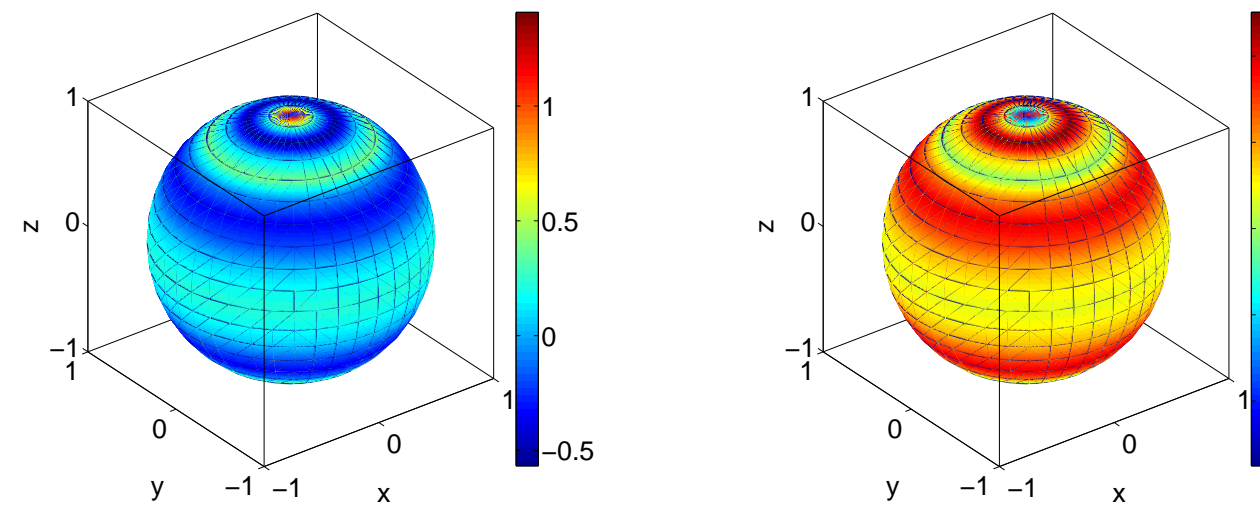

Figure 18: The far-field pattern $\psi_{\infty, \mathrm{FL}}$ of the scattered field from the uniformly distributed point scatterers in Example 3 via the Foldy-Lax formulation. (left) real part; (right) imaginary part.
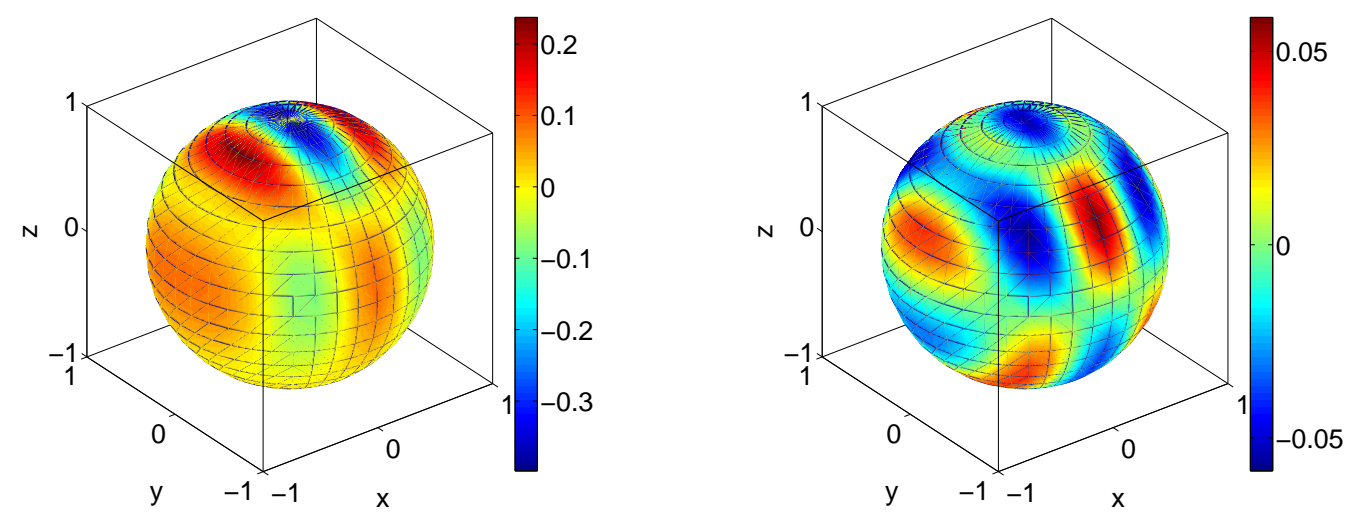

Figure 19: The far-field pattern $\psi_{\infty, \mathrm{E}}$ of the scattered field from the two spherical obstacle scatterers in Example 3 via the boundary integral equation. (left) real part; (right) imaginary part. 



Figure 20: The far-field pattern $\psi_{\infty, \mathrm{GFL}}$ of the scattered field from the uniformly distributed point scatterers and the two spherical obstacle scatterers in Example 3 via the generalized Foldy-Lax formulation. (left) real part; (right) imaginary part.
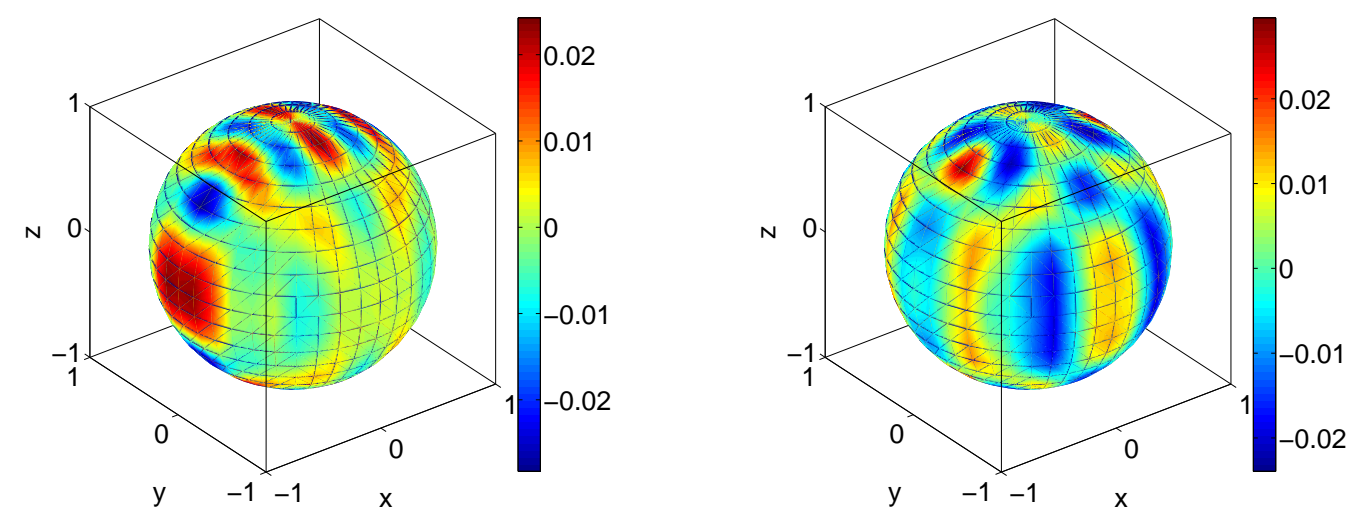

Figure 21: The difference of the far-field patterns $\psi_{\infty, \mathrm{GFL}}-\psi_{\infty, \mathrm{E}}-\psi_{\infty, \mathrm{FL}}$ from the uniformly distributed point scatterers and the two spherical obstacle scatterers in Example 3. (left) real part; (right) imaginary part.
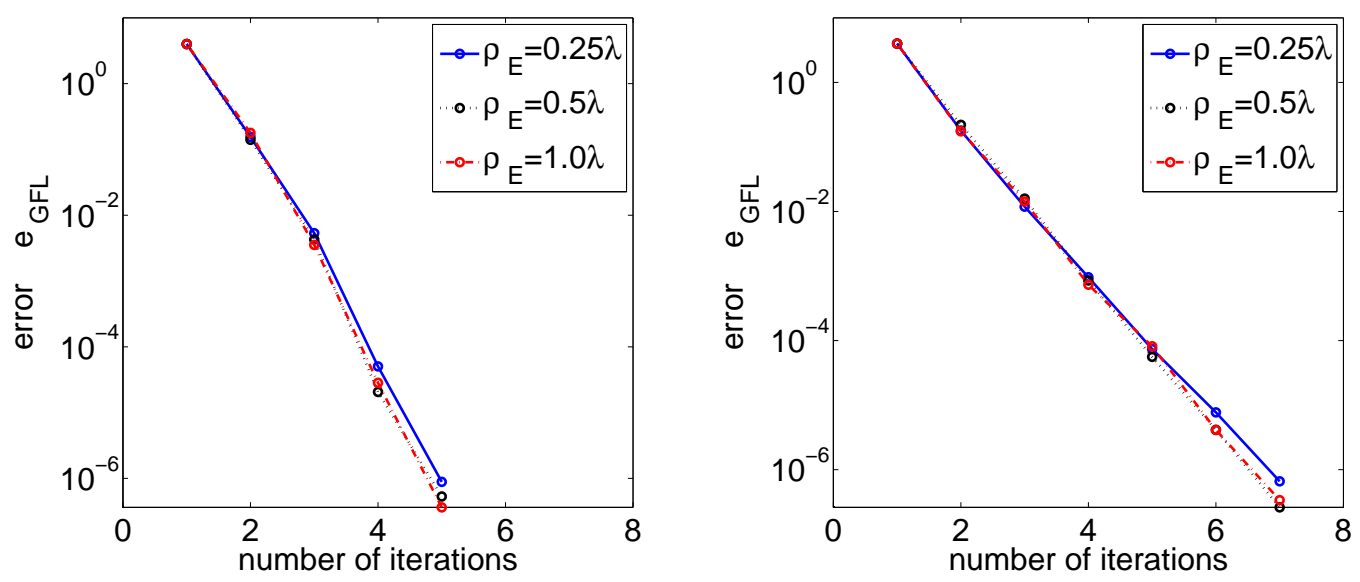

Figure 22: Error of two consecutive approximations using different separation distance for Example 3. (left) block Gauss-Seidel iteration; (right) block Jacobi iteration. 
physical parameters such as the scattering coefficient and the separation distances. Computationally, an efficient block Gauss-Seidel iterative method was proposed to solve the coupled system. At each step of iteration, only a linear system of algebraic equations or a boundary integral equation for a single obstacle scatterer needs to be solved. Sufficient condition for the convergence of the iteration is provided in terms of physical parameters. Convergence is investigated for the effects of the number of point scatterers, the scattering coefficients, and the separation distances through numerical experiments. We are intended to extend the proposed method to the three-dimensional Maxwell equations and will report the progress elsewhere.

\section{References}

[1] W. Chew, Waves and Fields in Inhomogeneous Media, Van Nostrand Reinhold, New York, 1990.

[2] D. Colton and R. Kress, Integral equation methods in scattering theory, John Wiley \& Sons, 1983.

[3] D. Colton and R. Kress, Inverse Acoustic and Electromagnetic Scattering Theory, SpringerVerlag, Berlin, 1998.

[4] L. Foldy, The multiple scattering of waves. I. General theory of isotropic scattering by randomly distributed scatterers, Phys. Rev. 67, 107-119 (1945).

[5] K. Huang and P. Li, A two-scale multiple scattering problem, Multiscale Model. Simul., 8 (2010), 1511-11534.

[6] K. Huang, K. Solna, and H. Zhao, Generalized Foldy-Lax formulation, J. Comput. Phys., 229 (2010), 4544-4553.

[7] R. Kress, Minimizing the condition number of boundary integral operators in acoustic and electromagnetic scattering, Q. Jl. Mech. Appl. Math. 38, 323-341 (1985).

[8] M. Lax, Multiple scattering of waves, Rev. Modern Phys. 23, 287-310 (1951).

[9] P. Martin, Multiple Scattering Interaction of Time-Harmonic Wave with N Obstacles, Cambridge University Press, 2006.

[10] J. Sun, S. Carney, and J. Schotland, Strong tip effects in near-field optical tomography, J. App. Phys. 102 (2007), 103103. 